\title{
Detection of endoscopic looping during colonoscopy procedure by using embedded bending sensors
}

This article was published in the following Dove Press journal:

Medical Devices: Evidence and Research

\section{Michael Bruce' \\ JungHun $\mathrm{Choi}^{2}$}

'Department of Mechanical Engineering, Ohio University, Athens, $\mathrm{OH}$, USA; ${ }^{2}$ Department of Mechanical Engineering, Georgia Southern University, Statesboro, GA, USA
Correspondence: JungHun Choi Department of Mechanical Engineering, Georgia Southern University, 20I COBA Drive, Statesboro, GA 30459, USA

$\mathrm{Tel}+19124784123$

Email jchoi@georgiasouthern.edu
Background: Looping of the colonoscope shaft during procedure is one of the most common obstacles encountered by colonoscopists. It occurs in $91 \%$ of cases with the N-sigmoid loop being the most common, occurring in $79 \%$ of cases.

Purpose: Herein, a novel system is developed that will give a complete three-dimensional (3D) vector image of the shaft as it passes through the colon, to aid the colonoscopist in detecting loops before they form.

Patients and methods: A series of connected links spans the middle $50 \%$ of the shaft, where loops are likely to form. Two potentiometers are attached at each joint to measure angular deflection in two directions to allow for 3D positioning. This 3D positioning is converted into a $3 \mathrm{D}$ vector image using computer software. MATLAB software has been used to display the image on a computer monitor. For the different configuration of the colon model, the system determined the looping status.

Results: Different configurations (N loop, reverse gamma loop, and reverse splenic flexure) of the loops were well defined using 3D vector image.

Conclusion: The novel sensory system can accurately define the various configuration of the colon during the colonoscopy procedure.

Keywords: colonoscopy, endoscopy, loop, 3D vector image, intubation

\section{Introduction}

Colonoscopy is the most effective procedure to investigate the gastrointestinal tract for colorectal disease. This is done by inserting an endoscope/colonoscope, with a camera fixed at one end, into the lower bowels through the anus. By closely inspecting the entire colon, the doctor can greatly reduce the risk for colorectal disease. However, this operation is not perfect and can be improved to be more efficient, safer, and easier on the patient. One obstacle that must be overcome during this procedure is looping of the colonoscope shaft. This can have a variety of effects ranging from extended procedure times, incomplete examinations, or even perforation of the colon wall. ${ }^{1}$ One study has shown that looping occurred in 91 out of 100 cases or $91 \%$ of the time. ${ }^{2}$ Looping increases operation time forcing the doctor to reposition the colonoscope by twisting and retracting the shaft. ${ }^{3}$ The longer the operation takes, the longer the patient is under anesthesia, which has its own risks associated with safety. It also increases pain and discomfort for the patient. By being able to know the position and orientation of the shaft inside the body, the doctor can more easily prevent dealing with loops that have formed and even prevent looping from occurring. ${ }^{4,5}$ The method discussed in this research will use a series of links connected inside the shaft that can calculate the posi- 
tion along intervals from the distal tip to the proximal end. These data can be used to then generate a three-dimensional (3D) vector image on a monitor for the doctor to observe.

There are some devices currently in use in the medical field to combat the problem of looping during the colonoscopy procedure. Each has had varying degrees of success. The four technologies that will be discussed include computerassisted colonoscopy, shape-locking guide, colonoscope overtube, and magnetic endoscopic imaging (MEI).

The first technology that will be discussed is the computerassisted colonoscopy system. The NeoGuide system utilizes a "fully articulated, computer-controlled insertion tube," which will allow each segment of the colonoscope to follow the same path that the tip has taken as the shaft is manually advanced into the colon. ${ }^{6}$ This significantly reduces looping and pain during the procedure. The NeoGuide system has had limited testing but with great results. Of the 10 patients in this study, the cecum was reached in all 10 cases with four of the cases showing disease or polyps. ${ }^{7,8}$

The next loop prevention method is a shape-locking guide. This device uses a shape-locking mechanism to prevent looping in the sigmoid section of the colon during colonoscopy. The shape-locking guide is an overtube that the colonoscope fits inside of. It can be changed from flexible to rigid by squeezing the handle when a rigid configuration is needed, such as in the sigmoid colon. When it becomes rigid, the colonoscope is then passed through the locked shape while preventing looping and large lateral forces on the wall of the colon. ${ }^{9}$

Related to the shape-locking guide is the overtube. The overtube is placed above the colonoscope to help eliminate excessive looping by increasing the overall stiffness of the colonoscope. A study was done to show the effects of an overtube on a conventional adult colonoscope and a pediatric scope. Pain was shown to decrease in nearly all cases, especially in the pediatric scope, and all but two of the cases showed completed colonoscopy. ${ }^{10}$ Another study was performed with an overtube on patients who had already had one incomplete colonoscopy. The cecum was reached successfully in $94 \%$ of the cases with a median time of 7 minutes to the cecum. ${ }^{11}$

The last loop prevention method to be discussed is MEI. MEI uses magnetism to provide a 3D image of the shaft of the colonoscope as well as the orientation and location of the distal tip in relation to the body. ${ }^{12}$ A study was carried out on the effect of using MEI in which endoscopists performed colonoscopies both when they used the MEI and when they did not. The study showed that "intubation times were shorter (11.8 vs 15.3 minutes), completion rates were higher (100\% vs $89 \%$ ), and the duration of looping was shorter (3 vs 5.4 minutes) when the endoscopist was able to see the imager view." 11,13
For the purposes of this study, a number of different designs were considered. The choices ranged from linear or angular sensors, to accelerometers, to cable mechanisms. The background for these technologies will be discussed in this section starting with the most promising of the ideas, the potentiometer.

Potentiometers, or pots, are variable resistors that can determine either linear or rotary displacement. They are used as rotary position sensors in a number of different applications. One study used a potentiometer to measure the angle of an ankle in motion. ${ }^{14}$ In this study, a digital protractor was used to calibrate a potentiometer which showed a linearity of 0.99 . The device was used for a smaller angle range of $110^{\circ}$. This was shown to accurately measure the angle. ${ }^{14}$ Another example used a potentiometer as a validation of a calculated angle for a tripolar total hip arthroplasty prosthesis. The potentiometer was connected to the joint and read the angular displacement with accuracy. ${ }^{15}$

The next possible design technology that could be used for this research is the $3 \mathrm{D}$ joint angle measurement system. This system describes a method to measure the relative position of a link and the corresponding angles in 3D. The study uses a rate gyroscope, accelerometer, and a magnetometer. ${ }^{16}$ By combining the accelerometer with both the gyroscope and the magnetometer, a more stable position can be found. This system is not dependent on a fixed reference, so it would be a great application for mobile objects. A system using this type of measurement would not need a series of links connected inside of the colonoscopy shaft; the sensor unit would only need to be placed at equal distances apart.

There are many examples of cable-driven systems in which cables can act as tensioned links to actuate parts of a system. One such system uses cables to help rehabilitate patients with impaired arms. ${ }^{17}$ The cables are attached to a solid structure that fits over the patient's arm and can be actuated by the use of motors. ${ }^{18}$ For the case of colonoscopy, a series of interconnected cables and links could be used to determine the angle of adjacent links and thus its 3D position. By knowing the change in length of the cable, an angle can be determined with respect to another link using simple trigonometric algorithms. One potential problem associated with this could be the amount of space that is available inside of the colonoscope. There will only be space for a limited number of cables which could limit the number of sensors and overall sensing length.

For the purposes of this study, a scaled up model will be used to show the functionality of the design. With that main goal, two objectives needed to reach this goal include designing and implementing both hardware and software. The hardware will deal with the rigid link system and use of sensors. The software will include data acquisition from 
the sensors and use of a data package to create a $3 \mathrm{D}$ vector image of the shaft. Along with these two main goals, there are a few design objectives/constraints that must be met.

\section{Methods}

In order to accomplish the slender body configuration as intended, both software and hardware are needed. With regard to hardware, many aspects have to be taken into account, including types of sensors, methods of installation, and so on. With regard to software, the type of software and the operating program to display the graphical image need to be chosen. The methods to choose and design all of these will be discussed in the following sections.

\section{Hardware}

The design of the hardware model includes the use of a potentiometer as an angular rotation sensor. The sensors are small enough to fit inside of the scope and have good reliability which would be good for the length of time needed for a procedure. The links would need to be connected together which could present some problems with the bending radius of the scope. The connected links and sensors would be able to determine the $3 \mathrm{D}$ position at multiple points along a length and could cover a large distance of the scope as well. It works by having a resistive element laid out between two pins. The third pin is located inside of these two pins and acts as the wiper that can move linearly or rotationally across the entire resistive element. By moving the wiper, the value of resistance between any two pins can be varied along the range of the upper and lower limits of the potentiometer. This can in turn vary the voltage from zero to the supply voltage. The potentiometer is widely used for doing such tasks as volume or sound control with a rotating knob. The potentiometer can also be used as a position or rotation measurement device. By knowing the resistance or voltage through the resistor, an angle can be calculated from calibrated tables and graphs. One article shows that potentiometers are used to both calibrate and measure the angle of an ankle joint. ${ }^{17}$ In another, a potentiometer was connected to a joint and used as an angular measurement device with very low error. ${ }^{18}$

The sensor that was chosen for this system is the Piher PT6KV-5K. It is a rotational type trimmer linear resistor. This trimmer resistor has a through hole for attachment. It is a single turn resistor with a maximum rotational angle of $235^{\circ}$ that is vertically adjustable. The resistance for the potentiometer varies from 0 to $5 \mathrm{k} \Omega$. The resistor has a maximum voltage of $100 \mathrm{~V} \mathrm{DC}$ with a nominal power of $0.1 \mathrm{~W}$. The potentiometer is $6.3 \mathrm{~mm}$ round with a height of $3.3 \mathrm{~mm}$.
Once it was decided that the potentiometer was the preferred sensor for this project, the next step was to determine how to integrate the sensor into a system to enable the recording of angular measurements. The most efficient way to connect the potentiometer to a system would be a simple joint such as a revolute so that it can measure the relative angle. The joints could then be connected with links of varying sizes.

A number of different types of joints were discussed as possible solutions. The first one was a spherical joint with two potentiometers attached to the rotating ball to measure rotation in different directions. Yu et al created a design where a spherical three degree of freedom active rotational ball joint was developed. ${ }^{19}$ His system had motors attached to the rotational ball, however, instead of potentiometers. Another joint discussed was the universal joint. This would allow for bending of the joint in two different directions while transferring rotational motion. There still exists a problem with attaching the potentiometers to the joint in such a way so as to measure the relative change of angle. The problem arises with the coupling connector, or the cross shaft, that allows bending in two directions. Since it is one piece, attaching two sensors to it that will measure two different rotations along with the space limitations make it impractical to use this design. However, the universal joint design could be modified to act as a pseudo-universal joint with the same rotational properties.

The design that was chosen behaves much the same as the universal joint without the rotational freedom. It consists of two pivot joints at a $90^{\circ}$ offset. The combination of these two joints will yield two degrees of freedom and allow for positioning at any point in three dimensions. The closer the two joints rotational axes are to each other the more the total joint behaves as a pseudo-universal joint. Ideally, these two joints should be at the same location but this is not possible for lack of space and decreased ability to conform to smaller radii. With the two bending axes and the use of some mathematics, distal positions can be found.

With the design needing to behave as a doubly pivoted joint, a system was developed that would mimic this closely. The ability to cover a large workspace and have a relatively small bending radius were two driving criteria for this design. If the model was not long enough, it would not be able to identify a loop. And if the joints were located at the same point, in order to cover the same area, the bending radius would have to be greatly increased. Because of these two points, bending radius and model length, the length between the sensor axes was changed to equidistant. This gives the ability to cover the same if not greater length. It also decreases 
the possible bending radius by shortening each individual link length. The model needed to be sturdy enough to handle forces from the colonoscope but slender enough to conform to a wide array of loops and configurations. A preliminary model was drawn up and tested.

Using this rendering, the first prototype was manufactured using simple materials. Figure 1 shows the first model that was built with the sensor attached along with a metric ruler for reference. The model consists of two links that are connected with a pin. It measures $25 \mathrm{~mm}$ in length with a diameter of $9 \mathrm{~mm}$. This module consists of two links attached with a sensor. The links were made out of 0.25 -inch wooden rod and machined down to specification using a vertical mill. The axle was made by trimming and flattening a small brad nail to fit into the cross-slot. The potentiometers will fit onto the axle with glue and adhere to the link adjacent to the axle using the leads to grip as well as glue. As the two connecting links rotate independent of each other, the axle will turn with one link, whereas the potentiometer will turn with another causing a relative angle to be measured.

This model proved to be quite effective in predicting the outcome when combined with the computer software; so, a new model was designed to elongate the total sensor length. A new manufacturing method was also looked into to produce the new model with significantly less machining time. In addition, wood was suitable for the preliminary prototype but a light plastic or metal was looked into as more desirable as a possible material based on the medical application and light-weight necessities.

With the abilities of $3 \mathrm{D}$ printing machines increasing and the availability of these machines, the new model was designed with the idea that the 3D printer could print the model with just as much if not more intricacy while decreasing the build time dramatically. With these new factors, a second, slimmer model was designed to further increase the sensor length. This model is shown in Figure 1.

The new model consists of two links that are connected with a pin. It measures $45 \mathrm{~mm}$ in length with a diameter of $7.5 \mathrm{~mm}$. For this new model, a fifth generation MakerBot

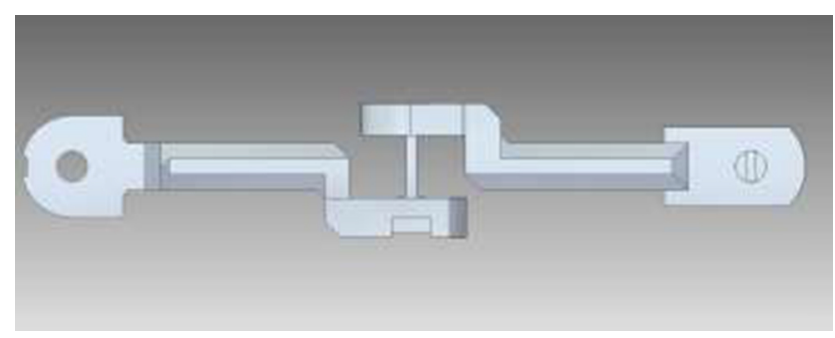

Figure I Single-joint module with two links showing two axes of rotation.
Replicator was used for the manufacture of the link system. When comparing the build times for both models, the MakerBot could build at least 5-10 times as many parts in the same amount of time. Figure 2 shows the MakerBot printing four sets of parts simultaneously layer by layer.

The base layer acts as a foundation between the build plate and the extruder. The inner layers of each part make up a honeycomb type shape for strength as well as limiting the amount of material used. The extruder (gold metal tip) can be seen building the first set of pieces for a test model. After the MakerBot was finished with the pieces, they could be easily separated from the base layer and cleaned of any excess material.

The process to make the axle was very similar to the previous model. A small brad nail was used after being cut, flattened, ground, and trimmed. First, the nail was cut to 4 mm length. Next, a vice was used to squeeze the body of the nail. This would act as the rectangular axle. After the body was flattened, a grinder was used to relieve the excess material to insure the nail would fit into the sensor's groove.

When all the pins were done they were glued into the links they were arranged in sets. The pins point in two directions along the entire length of the scope. This gives the sensors two axes of bending which are perpendicular to each other. Half of the sensors point in one direction, whereas the other half are offset by $90^{\circ}$. The potentiometers were fixed to the pins on each link with JB Weld, a cold weld two-part epoxy (JB Weld, Sulphur Springs, TX, USA). This epoxy provides a high-strength permanent bond and attaches to many different surfaces such as metal and plastic. After the epoxy had cured over the night, the model could be handled safely. Each joint was cleaned of any excess glue. Each joint was also tested to verify that no excess glue locked up the rotating axle of the potentiometer. Each sensor was glued correctly and no problems arose.

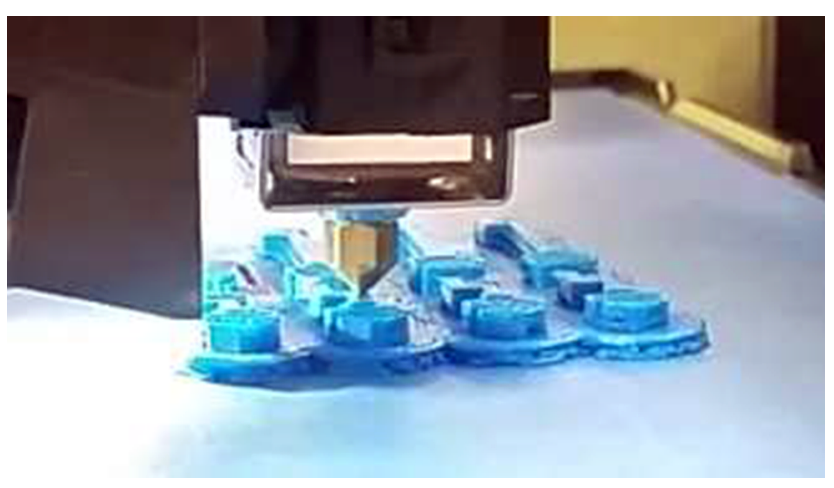

Figure 2 From this snapshot, the individual parts can be seen sitting on top of a base layer. 
The next step requires the leads of the potentiometers to be connected together with the thin signal wire. A soldering iron was used to painstakingly attach the wires firmly to the positive, negative, and signal leads. Each sensor is attached in parallel to the $5 \mathrm{~V}$ power supply and $0 \mathrm{~V}$ ground. After each sensor was wired, a voltmeter was used to confirm that a voltage was measurable across the signal pin to prove the soldered wire made a complete connection. Then, the output signal wires were attached to the wiper pin on the potentiometer. To prevent the wires from detaching, liquid insulation was applied generously to each solder point. This gives extra protection both from tearing the solder out and from another wire connecting and altering the signal. Each of the signal wires was then hooked into the data acquisition device. This device processes voltage signals and stores them in a file. The file can then be used in a computer program to generate an image.

\section{Kinematics theory and equations}

This section focusses on all the necessary equations and methods of solving 3D positioning for this project. Also discussed is the method that is used to determine accuracy of the loop configuration found from the sensor output.

\section{Transformation matrices}

Knowing the relative angles between the links is a very useful piece of information, but that alone would not be able to generate a complete image. In order to know the distal tip position, each sensor position must also be known with respect to the proximal tip (origin). This is, in effect, forward pose kinematics and can be done with a series of transformation matrices. The transformation matrix augments the original axes by multiplying by the angle of deviation with respect to the original. This matrix can only handle a rotation about a single axis at a time; so, to accommodate all 32 links, there are just as many matrices. Figure 3 shows the transformation of a single set of axes.

As can be seen from Figure 3, a link of length $L_{0}$ that originates at the origin $P$ and travels to $P_{0}$ shows a $\mathrm{Z}$ axis transformation. The new axes, $X_{0}$ and $Y_{0}$, have rotated an equal amount and have remained orthogonal with $Z_{0}$. This can be represented by the matrix shown below in Equation 1 .

$$
\left[\begin{array}{l}
P_{x 0} \\
P_{y 0} \\
P_{z 0}
\end{array}\right]=\left[\begin{array}{ccc}
\cos \left(\theta_{0}\right) & \sin \left(\theta_{0}\right) & 0 \\
-\sin \left(\theta_{0}\right) & \cos \left(\theta_{0}\right) & 0 \\
0 & 0 & 1
\end{array}\right] *\left[\begin{array}{c}
P_{x} \\
P_{y} \\
P_{z}
\end{array}\right] .
$$

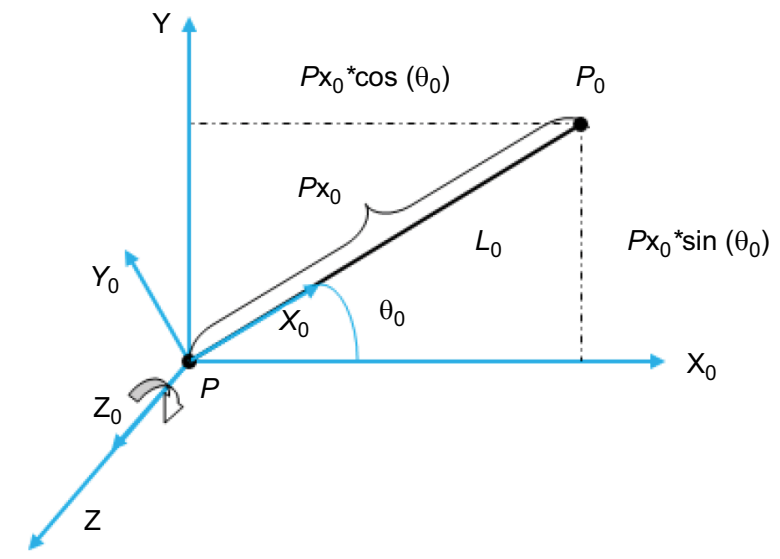

Figure 3 Example of an axis transformation is shown about the $\mathrm{Z}$ axis. The $\mathrm{X}$ and $Y$ axes rotate an angle of $\theta$.

In this equation, the values for $\mathrm{P}_{\mathrm{x} 0}, \mathrm{P}_{\mathrm{y} 0}$, and $\mathrm{P}_{\mathrm{z} 0}$ represent the 3D position of point $P_{0}$ with respect to the newly formed set of axes. The subscripted variables $P_{x}, P_{y}$, and $P_{z}$ are the positions of the point of origin $\mathrm{P}$ with respect to the original set of axes. Using this transformation matrix, the position of point $\boldsymbol{P}_{\mathbf{0}}$ can be found from the vector $\overline{\boldsymbol{P P}_{0}}$. The vector starts at $\boldsymbol{P}$ and ends at $\boldsymbol{P}_{\mathbf{0}}$. This all happens in the newly formed direction of $\boldsymbol{X}_{0}$. This vector can be written as follows:

$$
\overline{P P_{0}}=L_{0} * P_{x 0} .
$$

Combining this with the matrix from Equation 1, the new vector can be expressed as:

$$
\overline{P P_{0}}=L_{0} *\left[\cos \left(\theta_{0}\right) * P_{x}+\sin \left(\theta_{0}\right) * P_{y}\right] .
$$

This gives the 3D position in terms of the original set of axes. Applying this with two transformations as in the twojoint module gives an expression for the position at the end of two links. Figure 4 shows the two-joint module kinematic diagram with axes transformations.

This figure only shows two links of the model, but due to the nature of the design, this diagram will repeat every two links along the entire length. There are only two transformations. The first rotation is a $\mathrm{Z}$ axis transformation, and the second rotation is a $\mathrm{Y}$ axis transformation. The position of point $P_{1}$ can be represented by $\overline{P P_{1}}$. This can be broken into two separate vectors as follows:

$$
\overline{P P_{1}}=\overline{P P_{0}}+\overline{P_{0} P_{1}}
$$




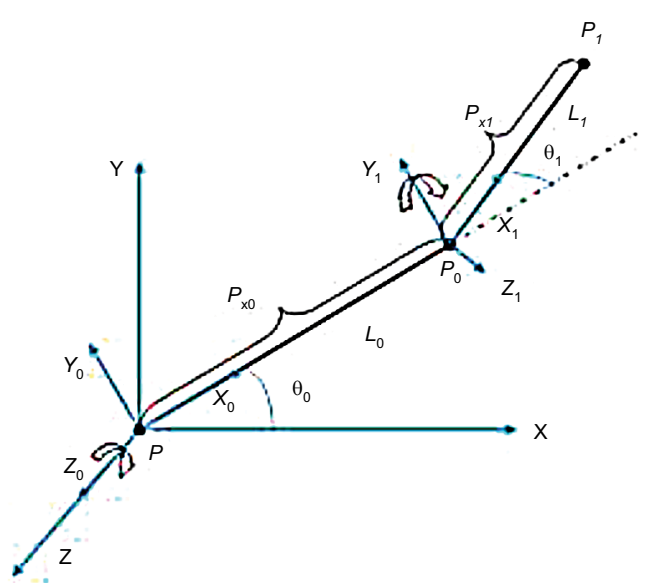

Figure 4 A kinematic diagram of a two-joint module shows two different axis transformations. The first is a $Z$ axis transformation followed by the $Y$ axis.

This can then be written with respect to particular axes.

$$
\overline{P P_{1}}=L_{0} *\left[\cos \left(\theta_{0}\right) * P_{x}+\cos \left(\theta_{0}\right) * P_{y}\right]+L_{1} * P_{x 1} .
$$

To find $P_{x 1}$, the second transformation matrix is needed. It is a $\mathrm{Y}$ axis transformation and has its own transformation matrix that can be seen in Equation 6.

$$
\left[\begin{array}{l}
P_{x 1} \\
P_{y 1} \\
P_{z 1}
\end{array}\right]=\left[\begin{array}{ccc}
\cos \left(\theta_{1}\right) & 0 & -\sin \left(\theta_{1}\right) \\
0 & 1 & 0 \\
\sin \left(\theta_{1}\right) & 0 & \cos \left(\theta_{1}\right)
\end{array}\right] *\left[\begin{array}{c}
P_{x 0} \\
P_{y 0} \\
P_{z 0}
\end{array}\right] .
$$

By combining and substituting Equation 1 in Equation 6, the second point $P_{2}$ can be expressed in terms of the original axes. This becomes

$$
\begin{aligned}
& {\left[\begin{array}{c}
P_{x 1} \\
P_{y 1} \\
P_{z 1}
\end{array}\right]=\left[\begin{array}{ccc}
\cos \left(\theta_{1}\right) & 0 & -\sin \left(\theta_{1}\right) \\
0 & 1 & 0 \\
\sin \left(\theta_{1}\right) & 0 & \cos \left(\theta_{1}\right)
\end{array}\right] *} \\
& {\left[\begin{array}{ccc}
\cos \left(\theta_{0}\right) & \sin \left(\theta_{0}\right) & 0 \\
-\sin \left(\theta_{0}\right) & \cos \left(\theta_{0}\right) & 0 \\
0 & 0 & 1
\end{array}\right] *\left[\begin{array}{c}
P_{x} \\
P_{y} \\
P_{z}
\end{array}\right] .}
\end{aligned}
$$

By finding $P_{x 1}$ from this equation and inserting it into Equation 5, the total solution for the position of $P_{2}$ can be found in Equation 8.

$$
\begin{aligned}
& \overline{P P_{1}}=L_{0} *\left[\cos \left(\theta_{0}\right) * P_{x}+\cos \left(\theta_{0}\right) * P_{y}\right]+ \\
& L_{1} *\left[\begin{array}{c}
\cos \left(\theta_{1}\right) * \cos \left(\theta_{0}\right) * P_{x}+\cos \left(\theta_{1}\right) \sin \left(\theta_{0}\right) * \\
P_{y}-\sin \left(\theta_{1}\right) * P_{z}
\end{array}\right] .
\end{aligned}
$$

Further simplifying this by combining the terms that have the position variables in common, the final vector form is Equation 9:

$$
\begin{aligned}
& \overline{P P_{1}}=\cos \left(\theta_{0}\right) *\left\{L_{0}+L_{1} * \cos \left(\theta_{1}\right)\right\} * P_{x}+ \\
& \left\{L_{0} * \cos \left(\theta_{0}\right)+L_{1} * \cos \left(\theta_{1}\right) \sin \left(\theta_{0}\right)\right\} * P_{y}-\left\{\sin \left(\theta_{0}\right)\right\} * P_{z} .
\end{aligned}
$$

This is done for each link in the entire model giving an accurate vector position of each link with reference to the original XYZ coordinate system. This can also be described as the $\mathrm{XYZ}$ coordinate of each point (sensor) along the length of the model.

In the full model, the matrix transformation is applied 32 times, once for each sensor. If this process is repeated for the second module set, the equations would follow Equation 10

$$
\begin{aligned}
& {\left[\begin{array}{l}
P_{x 3} \\
P_{y 3} \\
P_{z 3}
\end{array}\right]=\left[\begin{array}{ccc}
\cos \left(\theta_{3}\right) & 0 & -\sin \left(\theta_{3}\right) \\
0 & 1 & 0 \\
\sin \left(\theta_{3}\right) & 0 & \cos \left(\theta_{3}\right)
\end{array}\right] *} \\
& {\left[\begin{array}{ccc}
\cos \left(\theta_{2}\right) & \sin \left(\theta_{2}\right) & 0 \\
-\sin \left(\theta_{2}\right) & \cos \left(\theta_{2}\right) & 0 \\
0 & 0 & 1
\end{array}\right]\left[\begin{array}{ccc}
\cos \left(\theta_{1}\right) & 0 & -\sin \left(\theta_{1}\right) \\
0 & 1 & 0 \\
\sin \left(\theta_{1}\right) & 0 & \cos \left(\theta_{1}\right)
\end{array}\right] *} \\
& {\left[\begin{array}{ccc}
\cos \left(\theta_{0}\right) & \sin \left(\theta_{0}\right) & 0 \\
-\sin \left(\theta_{0}\right) & \cos \left(\theta_{0}\right) & 0 \\
0 & 0 & 1
\end{array}\right] *\left[\begin{array}{l}
P_{x} \\
P_{y} \\
P_{z}
\end{array}\right] .}
\end{aligned}
$$

In this equation, the subscripts 2 and 3 correspond to the next links in the system. By expanding this out fully, $P_{x 3}$ which is the variable that represents the distance along the axis between $\mathrm{P}_{2}$ and $\mathrm{P}_{3}$ can be found. This is obtained in Equation 11.

$$
\begin{aligned}
& P_{x 3}=\left(\begin{array}{l}
\cos \left(\theta_{0}\right) \cos \left(\theta_{1}\right) \cos \left(\theta_{2}\right) \cos \left(\theta_{3}\right)- \\
\cos \left(\theta_{0}\right) \sin \left(\theta_{1}\right) \sin \left(\theta_{3}\right)-\sin \left(\theta_{0}\right) \sin \left(\theta_{2}\right) \cos \left(\theta_{3}\right)
\end{array}\right) * \\
& P x+\left(\sin \left(\theta_{0}\right) \cos \left(\theta_{1}\right) \cos \left(\theta_{2}\right) \cos \left(\theta_{3}\right)-\sin \left(\theta_{0}\right) \sin \left(\theta_{1}\right)\right. \\
& \sin \left(\theta_{3}\right)+\cos \left(\theta_{0}\right) \cos \left(\theta_{3}\right) \sin \left(\theta_{2}\right) * P y+\left(-\cos \left(\theta_{2}\right) \cos \left(\theta_{3}\right)\right. \\
& \left.\left.\sin \left(\theta_{1}\right)-\cos \left(\theta_{1}\right) \sin \left(\theta_{3}\right)\right) * P z\right) .
\end{aligned}
$$

The full kinematic model diagram can be seen in Figure 5. The middle portion has been removed due to redundancy but the beginning and ending sections show the numbering and notation of each link and its appropriate transformation. The figure shows four separate axis transformations at the start and four axis transformations at the end. 


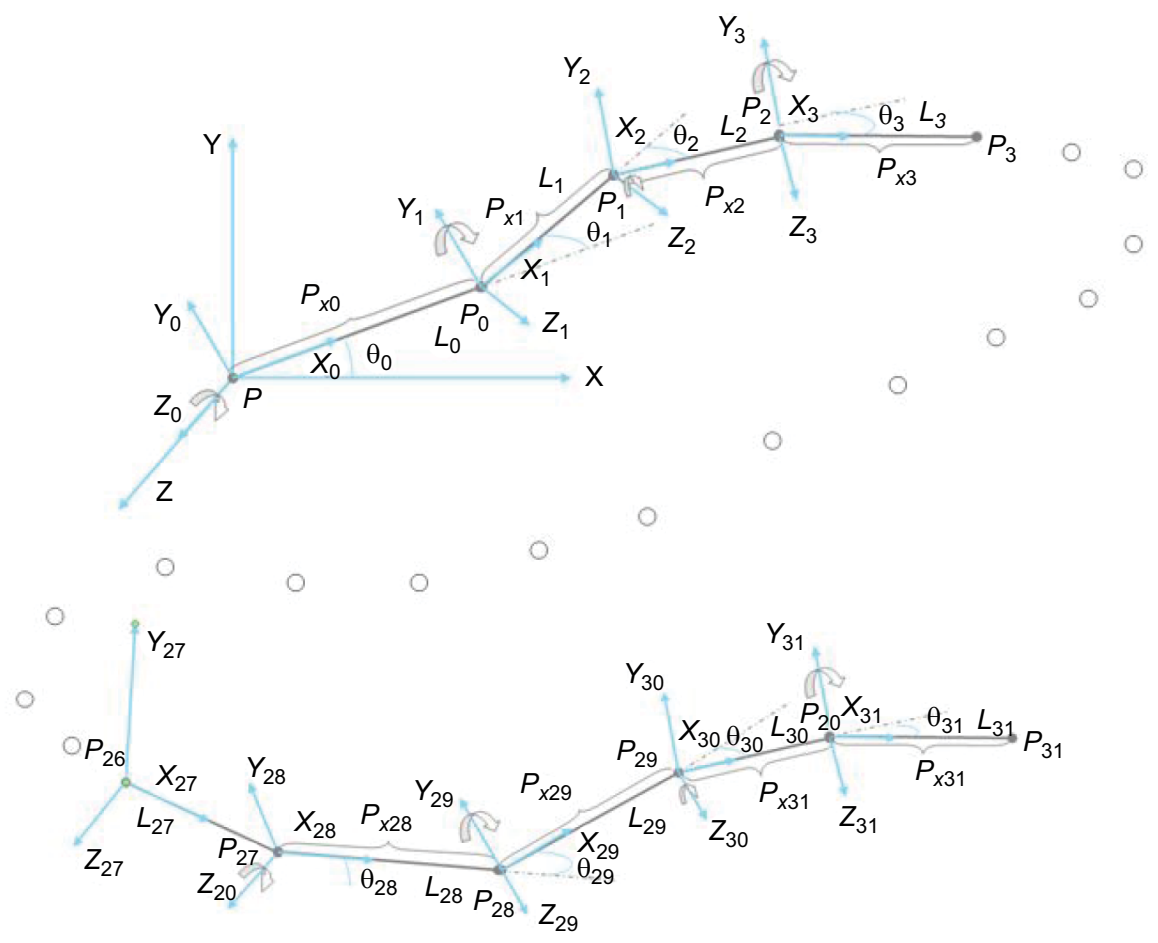

Figure 5 Total kinematic diagram excluding inner segments with repetitive nature. Four axis transformations are shown beginning (above) and ending (below).

To avoid extremely large matrix equations, MATLAB (MathWorks, USA) was used to calculate all the sensor positions. For each point, a rotation matrix was multiplied by the previous points' position according to Equation 6 . These values were used to calculate the $3 \mathrm{D}$ value of $P_{x n}$ for every sensor. This value was split into three parts corresponding to $\mathrm{P}_{x}, \mathrm{P}_{\mathrm{y}}$, and $\mathrm{P}_{\mathrm{z}}$. These corresponding numbers are equal to the $\mathrm{X}, \mathrm{Y}$, and $\mathrm{Z}$ components of the desired point relative to the original set of axes. Once all these positions were calculated, the $\mathrm{X}, \mathrm{Y}$, and $\mathrm{Z}$ coordinates were summed with all previous points to find the $3 \mathrm{D}$ position of any desired point. These matrix equations are located in the MATLAB code and are the backbone of the positioning calculations.

\section{Looping accuracy}

The output from the sensors will be used along with a computer program to generate a $3 \mathrm{D}$ vector image that will display on the screen. This will be able to show the configuration using the equations from the "Kinematics theory and equations" section. These images will be compared to the actual configuration of the model using equations that will be explained in this section.

To find the actual configuration of the model, a piece of string equal to the length of the model will be marked at equal distances along the length. This string will then be laid on the colon tube configuration with points of the string corresponding to particular sensors in the link model. Each point's 3D position will be found using a ruler and square relative to the origin. These coordinates will then be compared to the coordinates produced from the MATLAB computer program.

Every point recorded for the true path will correspond to a similar point on the predicted path. Connecting these points will give a segmented line that will model the configuration. By doing this for both the true and predicted paths, a method for comparing the two arises.

Splitting up the original curve into multiple piecewise segments allows a simple angular comparison to be made. Each predicted vector will be compared with its counterpart true vector. This will be done by comparing the angular deviation between the two. If the two vectors are parallel, then the angle between them will be zero. This method is suitable as a comparison for this model because the overall 3D position does not need to match exactly the true position. The ultimate goal is to compare the paths or trajectories of the curves. If each individual vector is similar but offset by a certain distance, then the model is still considered a success because the shapes will match.

This can be explained more thoroughly with the help of equations and figures. Consider the vectors for each path in the model shown in Figure 6. 


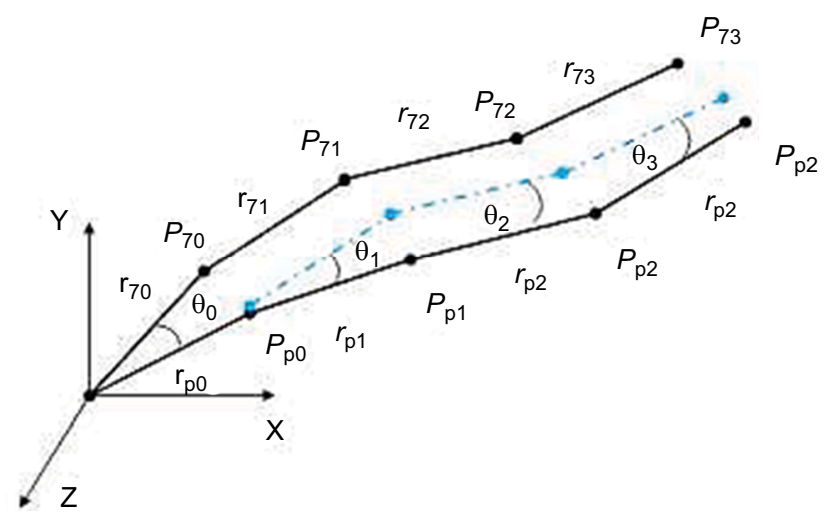

Figure 6 Two vectors shown starting at the origin and ending at points.

Each point P's coordinates are found by either measuring them or as output from the computer software. To compare these two vectors $r_{p}$ and $r_{T}$, the dot product equation (Equation 12) is used

$$
r_{T} \cdot r_{P}=\left|r_{T}\right|\left|r_{P}\right| * \cos (\theta)
$$

According to this equation, the dot product is equal to the product of the magnitude of each vector $\left|r_{T}\right|\left|r_{P}\right|$ multiplied by the angle existing between both vectors. Rearranging this equation, the angle can be found and is shown in Equation 13:

$$
\theta=\arccos \left(\frac{r_{T} \bullet r_{P}}{\left|r_{T}\right|\left|r_{P}\right|}\right)
$$

The dot product of each vector can be simply represented by the multiplication of each vectors $\mathrm{X}, \mathrm{Y}$, or $\mathrm{Z}$ coordinates, and then summed to give a single answer. If a single vector is represented by Equation 14,

$$
r_{T}=x_{T} \hat{i}+y_{T} \hat{j}+z_{T} \hat{k}
$$

then the dot product can be represented as follows:

$$
r_{T} \cdot r_{P}=x_{T} \hat{i} * x_{P} \hat{i}+y_{T} \hat{j} * y_{P} \hat{j}+z_{T} \hat{k} * z_{P} \hat{k}
$$

The magnitude of the vector is defined as the square root of the sum of each individual component squared and is shown in Equation 16.

$$
\left|r_{T}\right|=\sqrt{x_{T} \hat{i}^{2}+y_{T} \hat{j}^{2}+z_{T} \hat{k}^{2}}
$$

Combining all these equations into Equation 13 gives the final result for the angle between two $3 \mathrm{D}$ vectors.

$$
\theta=\arccos \left(\frac{x_{T} \hat{i} * x_{P} \hat{i}+y_{T} \hat{j} * y_{P} \hat{j}+z_{T} \hat{k}^{*} z_{P} \hat{k}}{\sqrt{x_{T} \hat{i}^{2}+y_{T} \hat{j}^{2}+z_{T} \hat{k}^{2}} * \sqrt{x_{P} \hat{i}^{2}+y_{P} \hat{j}^{2}+z_{P} \hat{k}^{2}}}\right) .
$$

By comparing the angle between the two vectors, a tangible error can be calculated. To compare the entire model to the real curve, the model is split up into 11 equal length vectors. Each of these vectors is then compared with the corresponding vector on the real path. These values will be used to calculate an overall average error for the given experiment.

This value of error is a measure of how the generated curve's shape matches the real curves'. The maximum allowable error for this value can be calculated by inspecting the colon model tube as shown in Figure 7.

The two vectors being compared $r_{T}$ and $r_{P}$ are the true and predicted path components, respectively, and are shown in Figure 7. The colon model wall thickness is the variable $t$, and $\theta$ is the angular error in degrees. The configuration above shows the maximum allowable deviation from the true path. This can be calculated with the given lengths and colon model wall thickness. The maximum angular deviation is found as shown in Equation 18.

$$
\theta_{\max }=\sin ^{-1}\left(\frac{t}{r_{P}}\right)
$$

Given the thickness of the model wall (1.5 inches) and the length of the predicted vector $(6.3 \mathrm{~cm})$, the maximum angle can be calculated. This value is found to be $37^{\circ}$. Therefore, the average angular error for each loop should be below this value.

\section{Software}

The hardware can only give values to voltages that the sensors read during the experiment. In order to generate a 3D image, a software package needs to be utilized to process these signals into something that can be seen from the computer screen.

There are many different data acquisition packages that can use the sensor's output and turn it into something usable like a figure, image, or graph. Some examples of software

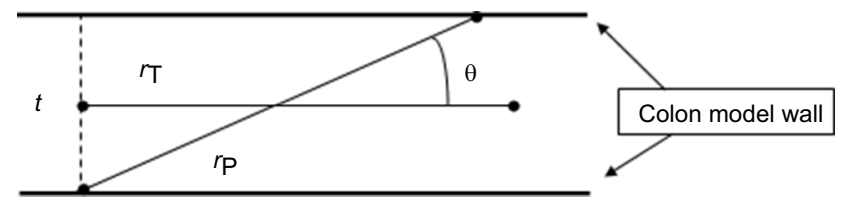

Figure 7 Maximum angular deviation in the worst case. 
packages include Vernier Lab Pro, WinDAQ Pro, LabVIEW, and MATLAB. These are just a few of the various packages available. For this project, a data acquisition device with a large number of channels is needed. The software must also be able to manipulate the data into a 3D image. Signal Express by LabVIEW was used to record the signal outputs from the model during operation. This signal can then be interpreted with different software.

For the prototypes and experiments performed, the computer program MATLAB was used to write code and generate $3 \mathrm{D}$ vector images. The signals from the model during operation were recorded in an Excel file. This file was then referenced from the MATLAB program. The generation of the image occurs after the collection of data and a single image or video is produced.

The MATLAB code used the transformation matrix approach and plotted each individual point along the model's path. This was done by inserting the matrices required for each links transformation as well as using a linear calibration curve to convert the incoming voltage into an angle in degrees. The individual $\mathrm{XYZ}$ coordinates of each sensor are then plotted along the entire curve. This can be done for a single snapshot of the configuration or for a set time where numerous consecutive data points are recorded.

\section{Experimental setup}

This subsection will give introductions to all the experiments performed for this study. The setup and procedures will be discussed at length and detail for each.

\section{Sensor calibration}

Before each sensor could be used in the prototype, it was tested to insure its reliability to a linear fit of values. The calibration was carried out simply by placing the sensor in a plain breadboard. The leads were attached to a DC power supply at $5 \mathrm{~V}$, and a voltmeter was used to measure the output voltage from the sensor. Once all the necessary equipment was setup and working properly, a polar grid was placed behind the sensor showing values of degrees from $0^{\circ}$ to $240^{\circ}$ in the clockwise direction. However, instead of starting at the typical $0^{\circ}$, the initial starting position for the wiper was at $180^{\circ}$ on the circle and it moved clockwise to $-40^{\circ}$ fulfilling the $240^{\circ}$ of rotation. In the next step, the voltage values were recorded every $10^{\circ}$ for each sensor. These numbers were recorded in a table and loaded into an Excel file to be analyzed. These values will give the relationship between sensor voltage and the angle of bend that is necessary for all other experiments.

\section{Model calibration}

After the sensors were calibrated, the model needed to be calibrated. This is due to small errors when manufacturing the axle pin. The axle is not necessarily at the starting 0 position. While in a straight line, it might be reading a value of $10^{\circ}-30^{\circ}$. This calibration is done by straightening each link by hand and recording the value output by the sensor. This value is considered as the new zero position and entered into the code to calibrate each particular sensor's angle equation. The original equation for the angle of each link is as follows:

$$
\theta(i)=\left(\frac{V(i)-v(i)}{-0.0239}\right)
$$

In this equation, $\theta(i)$ is the angle of the link, $V(i)$ is the voltage output from the sensor at the given angle, and $v(i)$ is the voltage at zero position. Using this equation will modify the existing program to more accurately identify looping.

\section{Looping analysis}

The main experiment of this study is the analysis of looping during the colonoscopy procedure. There are many different types of loops that can form as was previously stated. Some of the most common are the sigmoid N-spiral and alpha loop. Others to be discussed in this section will include some nontypical loops to show that the model can perform under varying circumstances. The two other types of loop formations to be studied are the reverse gamma loop and the reverse splenic flexure loop..$^{20,21}$

For all the loops that will be tested, a 1.5-inch diameter tube was used for the loop formations desired. The prototype model was then advanced into the loop while the system recorded the sensor data. Due to the corrugated nature of the colon tube as well as the prototype tube, a string has been attached to the front end of the model. This will be used to help advance the device if it meets with great resistance due to the corrugation. Once the end of the loop is reached, the experiment is stopped. This process will be repeated for a total of three tests per loop to show repeatability.

In order to compare the generated images' accuracy to the actual loop, the XYZ coordinates of multiple points need to be found once the loop is formed. To find these values, a string marked at equal distances is laid across the loop. An X, $\mathrm{Y}$, and $\mathrm{Z}$ coordinate grid is then projected onto the platform using a ruler and square. The position of each mark along the string is recorded in the $\mathrm{X}, \mathrm{Y}$, and $\mathrm{Z}$ dimensions. 


\section{Alpha loop}

The alpha loop is one of the common loops found in the sigmoid colon. ${ }^{4}$ The experimental setup can help explain the shape and function of the loop in Figure 8.

The model will enter the loop from the proximal hole and travel upward spinning back down and exiting at the distal end. The alpha loop has a 5-inch inner diameter and the entrance and exit are spaced $15 \mathrm{~cm}$ apart laterally.

\section{Sigmoid N-spiral}

The N-spiral loop is the most common loop in the sigmoid colon. ${ }^{4}$ It is similar to the alpha loop with a slightly varied ending position and angle of bend. The experimental setup for the N-spiral loop can be seen in Figure 9.

The N-spiral loop's radius of curvature for the first portion is 5 inches and increases slightly for the top radius. In this loop, the scope enters the colon at the lower opening and travels upward. Next, it will spin to the left and flatten out at a higher height than the entrance. Following this, it will begin to rise again and exit the loop at the distal opening in an upward trajectory.

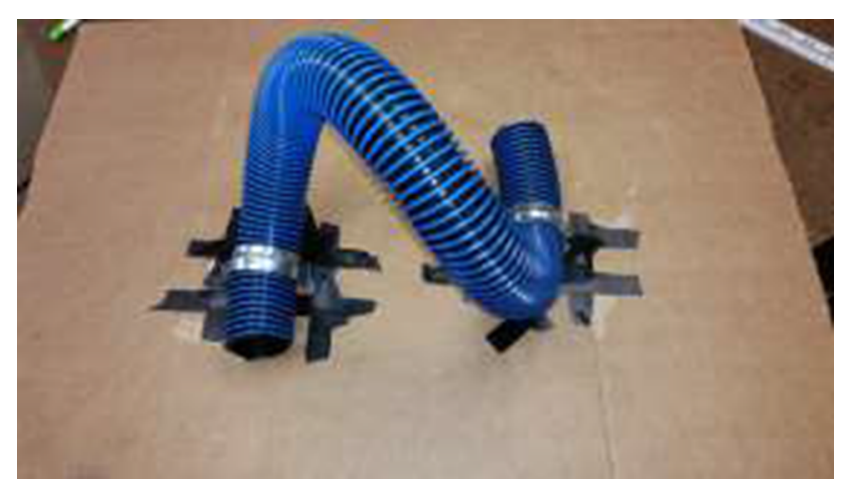

Figure 8 A small alpha loop configuration.

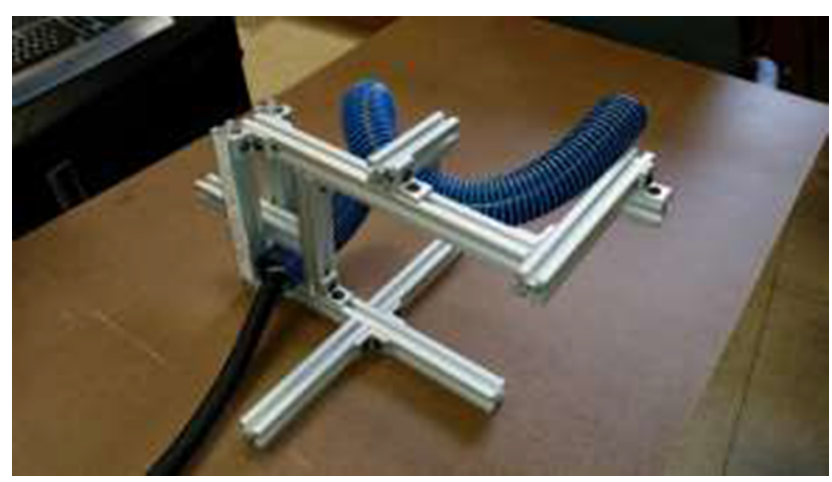

Figure 9 A sigmoid $\mathrm{N}$-spiral loop formed in a fixture awaiting the experiment.

\section{Reverse gamma loop}

The reverse gamma loop is a less common loop compared to the sigmoid $\mathrm{N}$-spiral and alpha loops. It resembles a small circle formed in the horizontal plane (Figure 10).

The reverse gamma loop has an inner diameter of 4 inches which is the minimum bending radius described in the design objectives. The upper colon loop segment sits directly on top of the lower portion making for a 1.5 -inch difference between the starting and finishing heights. The model enters the inferior opening and travels clockwise in the loop before exiting the upper opening in the same direction that it entered.

\section{Reverse splenic flexure loop}

The reverse splenic flexure loop is also an altogether uncommon loop formed in the ordinary colonoscopy procedure. It slightly resembles a reverse spiral loop. The experimental setup is shown in Figure 11.

In this configuration, the model will begin in the bottom portion before performing a vertical loop. It will then loop back upward but at a distance from the original loop. The primary loop has a radius of 2 inches and the secondary loop's radius constantly increases after exiting the first loop.

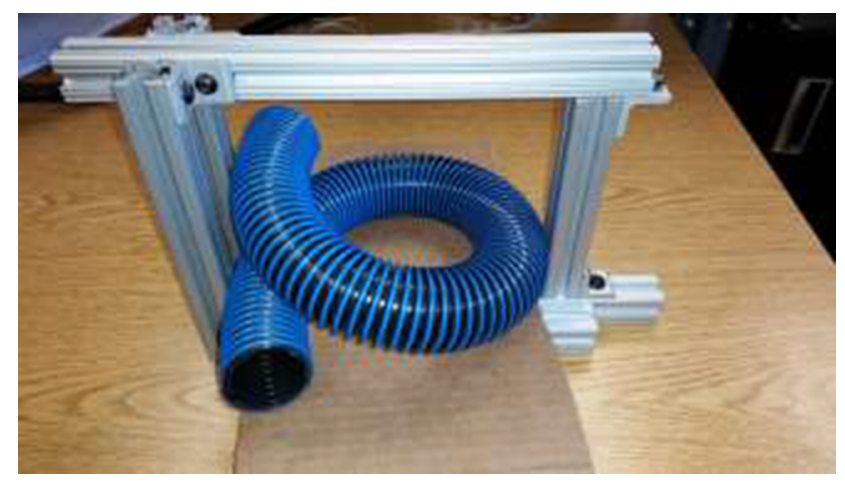

Figure $10 \mathrm{~A}$ tight reverse gamma loop is formed.

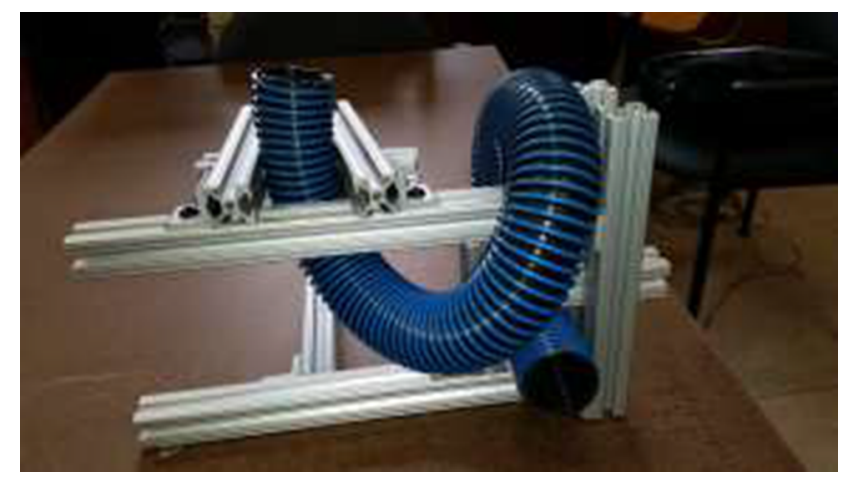

Figure I I A small reverse splenic flexure loop formed in the sigmoid colon. 


\section{Results}

Once all the experiments were completed and all the data were tabulated into tables and figures, they could finally be analyzed. This section will present the results from every experiment performed for this study including the calibration, stiffness test, loop analysis, and looping accuracy comparison.

\section{Sensor calibration}

The potentiometers were individually calibrated by recording the voltage at increments of $10^{\circ}$. The voltage values were then plotted versus the corresponding angles as shown in the figure. For each angle tested, there are 32 different values plotted in Figure 12.

The initial and final $30^{\circ}$ of the potentiometers range do not behave linearly. This means that as the resistance nears the ends of its mechanical rotation, it starts to flatten out and become constant. By utilizing the inner $180^{\circ}$, a trend line can be added with a coefficient of determination of 0.999 proving its linearity. The equation for the voltage as a function of angle of rotation is

$$
Y=-0.0239 X+5.4616 .
$$

In this equation, $Y$ is the voltage output and $X$ is the angle of rotation of the sensor.

\section{Initial prototype}

Once the calibration test proved successful, an angular measurement test was done to prove that the potentiometers could be used as a relative angle measurement sensor. Three links were hooked together attached with three potentiometers. The potentiometers were then fixed to the previous link with an alligator clip to ensure that the angle measured is relative to the previous link. It showed an angle of rotation of $40^{\circ}$ between each link. Once the angle between links was verified, the Vernier Lab Pro data acquisition (Vernier Software \& Technology, USA) package was used to measure the voltage from each potentiometer.

The voltage value from the calibration equation equals $4.563 \mathrm{~V}$. Comparing this to the values above shows that there is a very small percent difference of only $0.5 \%$. Figure 13 shows the link configuration plotted with MATLAB using the voltage readings, and the same lengths as the experimental setup.

Once this was proven to be successful and the initial prototype was completed, the model was wired together and attached to the system. The output signals were attached to

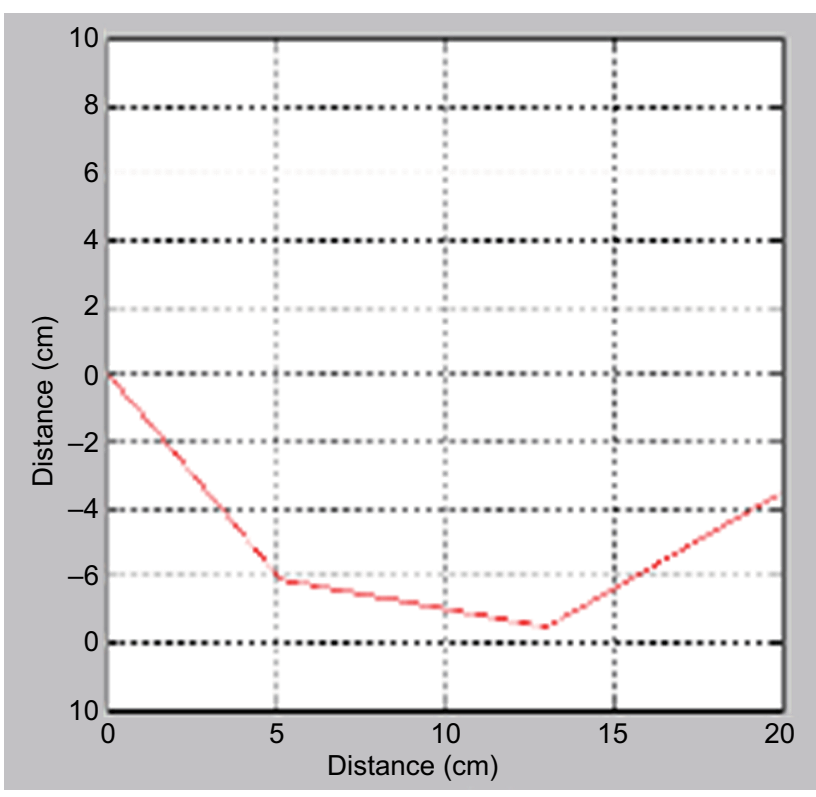

Figure 13 MATLAB code of three links each at a $40^{\circ}$ angle.

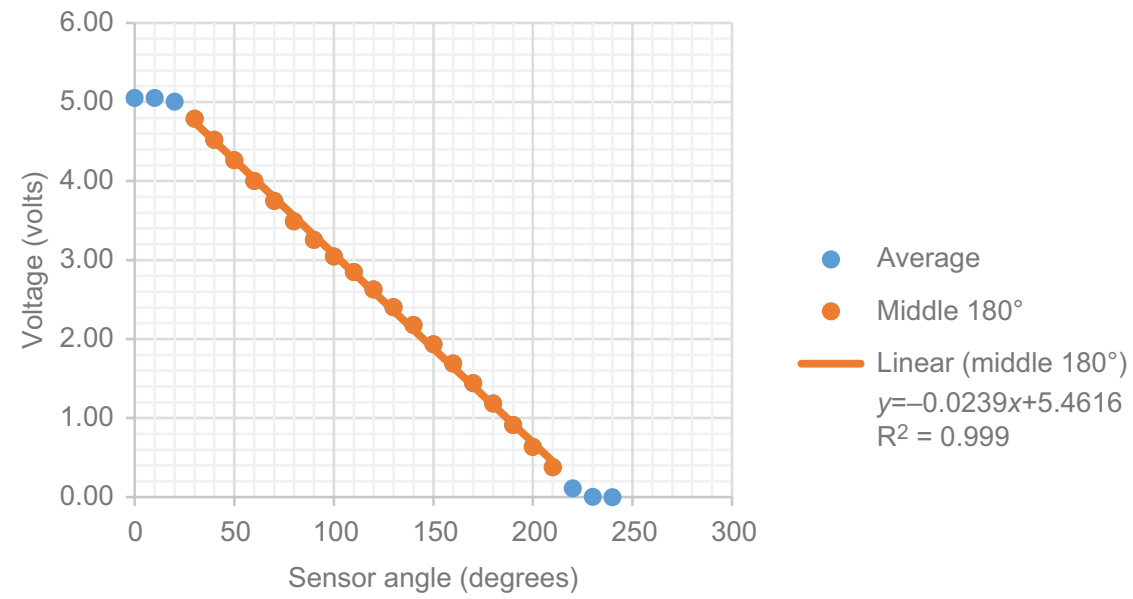

Figure 12 The average voltage for each given angle calculated and plotted, omitting the beginning and ending $30^{\circ}$ due to nonlinearity 
the data acquisition (DAQ) while the power and ground were wired to the power supply. A system diagram showing all components can be seen in Figure 14.

This experimental setup was used to collect data for two different configurations. The first configuration was a baseline reading to record the values of each sensor when the model was in a straight line. The second recording was done with a small alpha loop of radius $3 \mathrm{~cm}$. Both experimental setups can be seen in Figure 15.

The data from these configurations were recorded and used in the MATLAB program. This was then able to generate two vector images. These two images are shown in Figure 16.

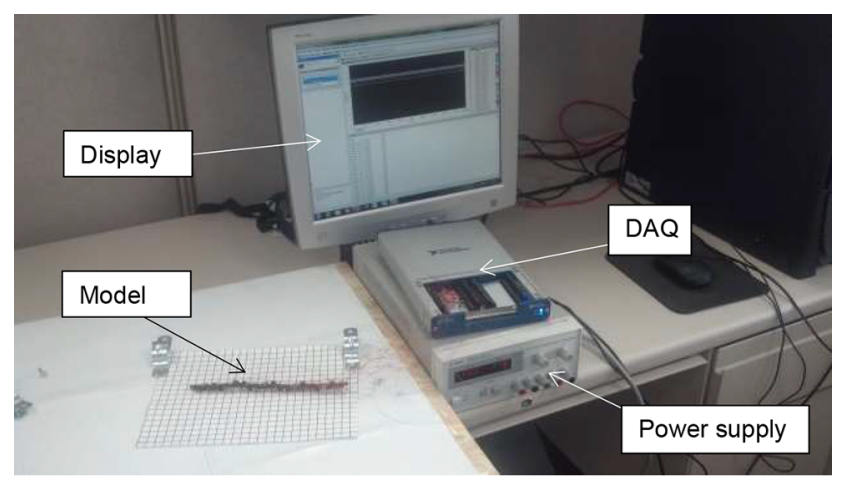

Figure 14 A complete system diagram showing the model wired with the DAQ and power supply, with the signal voltages output to the display. Abbreviation: DAQ, data acquisition.

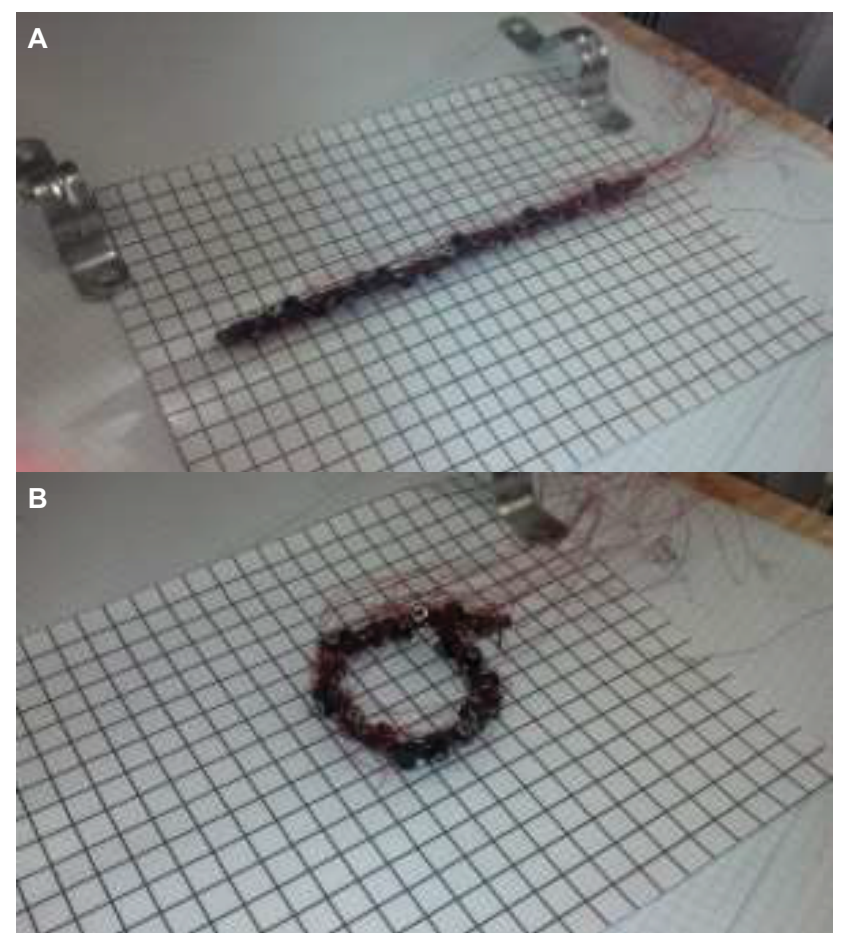

Figure 15 Model configurations in the experimental setup. (A) A straight line, base reading. (B) An alpha loop of $3-\mathrm{cm}$ radius.
As can be seen from this figure, the straight line and loop images match the original configuration exactly as should be expected.

\section{Final model testing}

Once the final model was assembled and ready to start taking measurements, the model needed to be calibrated. This was done as stated in the "Model calibration" section. Table 1 shows the starting position voltage for each sensor from the original calibration equation. It also has the values found experimentally from straightening each individual link. These newly modified values for the starting position voltage were added to the MATLAB program.

One additional problem encountered with the model occurred in some preliminary tests. The problem that arose showed the predicted configuration as incomplete, or at least it looked incomplete. All the sensors and links were plotted, but the generated image would stop short and only show maybe $75 \%$ of the actual curve. The reason for this was that there exists extra space inside of the colonoscopy tube for the sensors. When the tube contacts the wall, the sensor does not have to move until a certain amount of bend causes the link to rotate. This means that the sensors were underpredicting the actual angle of bend of the scope. This was dealt with using a small modification to the slope of the calibration data found empirically. Figure 17 shows the original calibration line and the modified line.

Multiple different modifications were tested until the final one was selected as the closest to the actual curve. Each different slope was tested in the image generation program. The value that most resembled the curve for each loop formation was the final modification. An example showing the incomplete curve followed by the modified curve is shown in Figure 18.

\section{Looping analysis}

All four types of loops, alpha, N-spiral, gamma, and reverse splenic flexure, were tested with overall success. For each experiment, there were three trials performed. The configuration of the loops was recorded every second for as long as it took to navigate through the entire section. This means that process of looping was visible, recorded, and in turn generated by the MATLAB computer program. This section will present these tests with figures and numerical comparisons alike. The figures will show the ending configuration of each loop only.

\section{Alpha loop}

The model was able to predict the alpha loop very well in both a visual test and the statistical analysis. Figure 19 shows the results from the alpha loop experiment. 

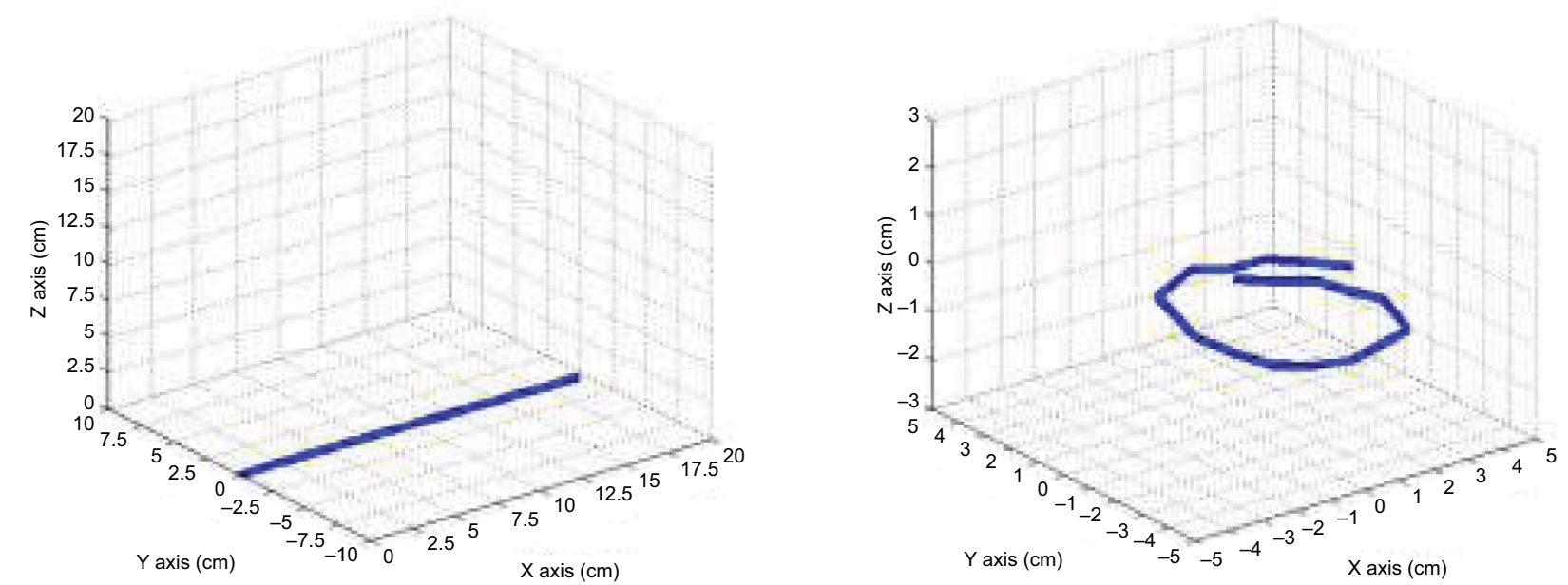

Figure 16 Computer-generated vector images of the configurations are shown. (A) A straight line. (B) A 3-cm alpha loop.

Table I Original versus modified calibration values

\begin{tabular}{llllll}
\hline Sensor & Original & Modified & Sensor & Original & Modified \\
\hline 1 & 2.32 & 2.31 & 17 & 2.33 & 2.22 \\
2 & 2.47 & 2.45 & 18 & 2.37 & 2.34 \\
3 & 2.42 & 2.29 & 19 & 2.50 & 2.5 \\
4 & 2.60 & 2.63 & 20 & 2.39 & 2.49 \\
5 & 2.41 & 2.32 & 21 & 2.81 & 2.77 \\
6 & 2.70 & 2.7 & 22 & 2.38 & 2.39 \\
7 & 2.18 & 2.17 & 23 & 2.45 & 2.42 \\
8 & 2.90 & 2.92 & 24 & 2.52 & 2.51 \\
9 & 2.30 & 2.23 & 25 & 2.48 & 2.39 \\
10 & 2.55 & 2.54 & 26 & 2.42 & 2.37 \\
11 & 2.65 & 2.51 & 27 & 2.35 & 2.34 \\
12 & 2.42 & 2.38 & 28 & 2.86 & 2.86 \\
13 & 2.52 & 2.53 & 29 & 2.38 & 2.32 \\
14 & 2.35 & 2.35 & 30 & 2.31 & 2.35 \\
15 & 2.32 & 2.29 & 31 & 2.44 & 2.46 \\
16 & 2.63 & 2.66 & 32 & 2.43 & 2.46 \\
\hline
\end{tabular}

Note: The modified values were found experimentally.

This figure presents the predicted path set forth by the sensor model from the MATLAB program. Three trial runs are shown. In the alpha loop, the model starts at the origin and moves straight along the positive $\mathrm{X}$ axis before moving upward in the positive $\mathrm{Z}$ axis all the while moving in the right direction along the $\mathrm{Y}$ axis. It flattens out at the top of the loop and begins to descend while reversing direction in the $\mathrm{X}$ direction. The multiple trials showed that the device is repeatable and can perform the same loop many times in succession. This can be compared with the true path, measured once the model had completed the loop, shown in Figure 20.

Comparing the diameters of the loops, the predicted loop shows about $18 \mathrm{~cm}$, whereas the true path shows about 15 $\mathrm{cm}$. The more telling comparison results from the vector to vector comparison explained in the "Hardware" section. The predicted and true paths were split into 11 equal length segments. The resulting comparison between these vectors is shown in Table 2.

From this table, the overall average angular deviation for any particular vector was $22.7^{\circ}$. This value is below the maximum allowable deviation of $37^{\circ}$. The lowest average for a trial occurred during the first trial, whereas the highest average occurred during trial 3 . Vectors 8 and 3 for every trial showed a considerably higher value which increased the averages higher than they might have been.

\section{Sigmoid N-spiral}

The sigmoid $\mathrm{N}$-spiral also proved to be accurately represented by the model. The output from the sensors correctly conforms to the true path. These images are shown in Figure 21.

The model shows a smooth curve that starts at the origin in the center of the graph and proceeds in the positive $X$ direction. It quickly moves upward in the $\mathrm{Z}$ direction, then levels off and moves laterally in the $Y$ direction. The end of the loop finished on another increase in the $\mathrm{Z}$ and $\mathrm{X}$ directions. The upward spiral shows about a $10-\mathrm{cm}$ diameter loop. This predicted path very closely resembles the true path shown in Figure 22.

The true path travels in much the same way as the predicted path and shows a similar upward spiral diameter of about $12 \mathrm{~cm}$. When looking at the individual vectors shown in Table 3, the visual test result is verified.

The total average for the experiment showed $23.8^{\circ}$ of deviation for any single vector compared to the true path. This value is also below the maximum error of $37^{\circ}$ and equals 

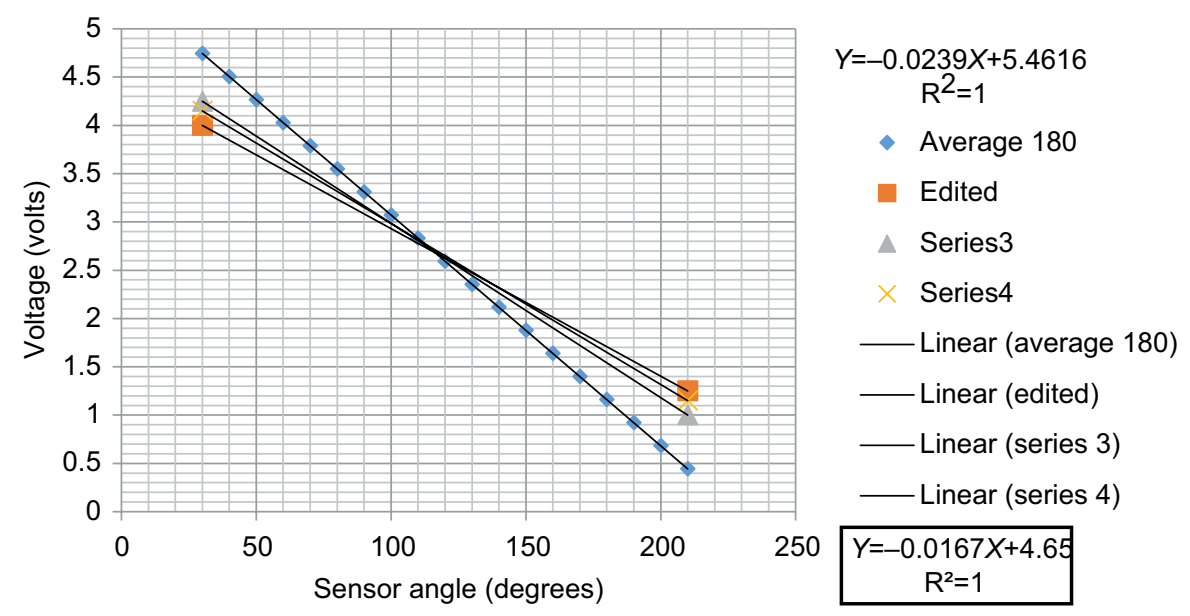

Figure 17 Newly calibrated voltage slope line.
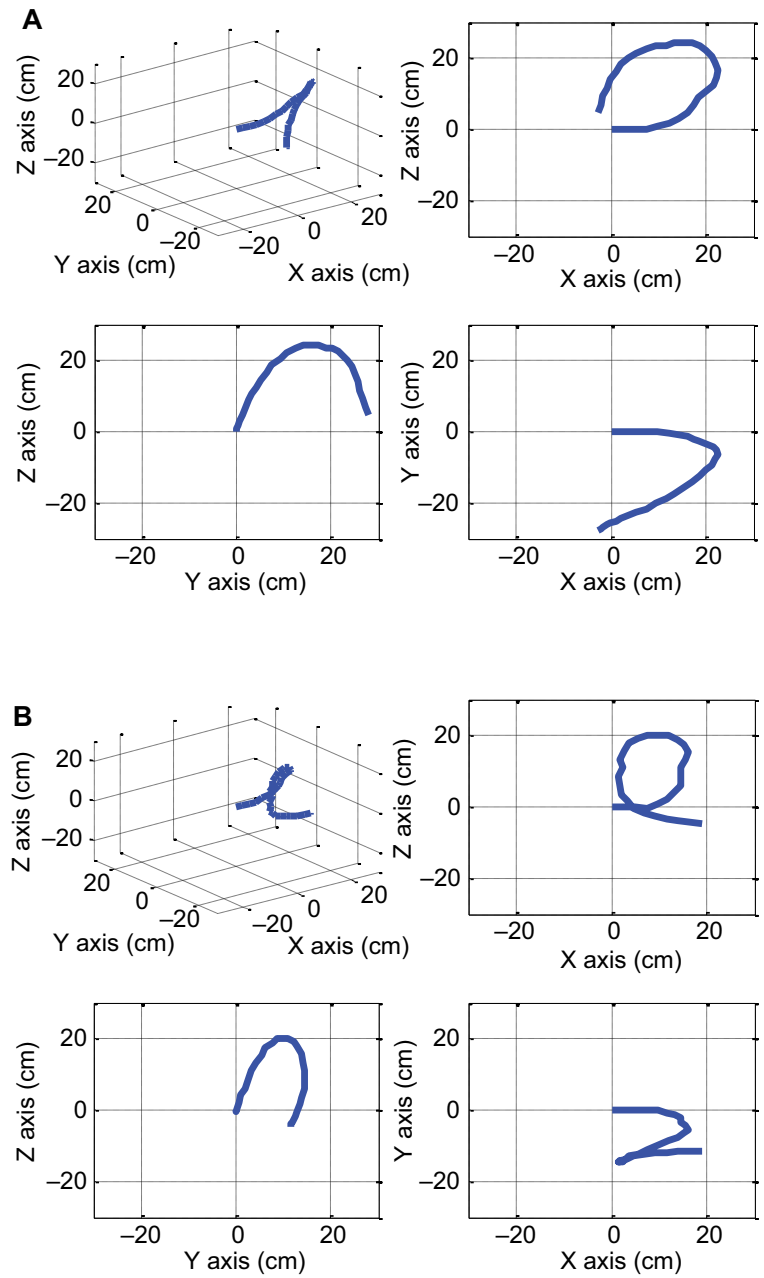

Figure 18 The original voltage equation produces an incomplete curve (A). The modified voltage equation completes the curve giving a more accurate representation (B).

$64 \%$ of the allowable error. The highest average numbered $26.6^{\circ}$, whereas the lowest totaled $21.1^{\circ}$. No single vectors stood out as being a possible outlier.

\section{Reverse gamma loop}

The reverse gamma loop proved to be the loop with the best approximation by the predicted path compared to the true path. The images for the reverse gamma loop are shown in Figure 23.

In the reverse gamma loop test, the model begins traveling in the positive $\mathrm{X}$ direction and makes a clockwise loop. The model increases and decreases in the $t$ axis as it travels clockwise around the colon model. Once the device reaches the opposite side of the loop, it begins to rise in the $\mathrm{Z}$ direction. The model finishes traveling on the positive $\mathrm{X}$ axis only completing the reverse gamma loop. The loop has a diameter of $15 \mathrm{~cm}$ with a positive difference in height of $8 \mathrm{~cm}$ from the start to the end of the loop. This matches very well with the true path shown in Figure 24.

The reverse alpha loop's true path is a near identical match to the predicted path. The diameter of the loop for the true configuration is also $15 \mathrm{~cm}$ with an increase in height of about $6 \mathrm{~cm}$ from start to finish. The only recognizable difference between the two is that the predicted path drops about $6 \mathrm{~cm}$ below zero in the $\mathrm{Z}$ direction before returning to the prescribed finishing position. The predicted path also finishes in a further $\mathrm{X}$ position but neither of these differences negatively affects the visual comparison. The incremental angular vector differences are given in Table 4.

The overall average angular deviation is $20.8^{\circ}$. This is below the error threshold and shows $56 \%$ of the maximum allowable error. The lowest for a single trial was shown to be $15.2^{\circ}$, whereas the highest deviation was $26.0^{\circ}$. Vectors 4 and 7 showed a slight increase compared to the rest of the vectors. These changes were seen in the differences mentioned above. 

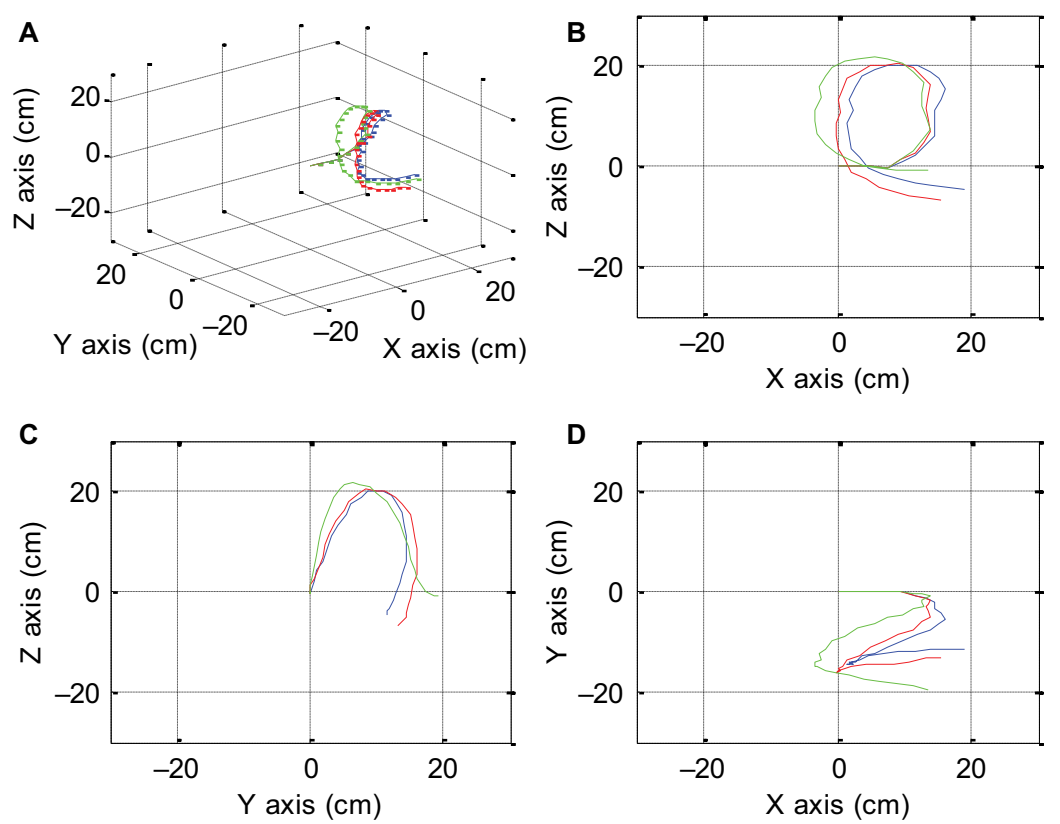

Figure 19 Multiple views of the predicted alpha loop test trials are shown. Isometric view (A), right-side view (B), left-side view (C), top view with right side as $X$ axis (D).
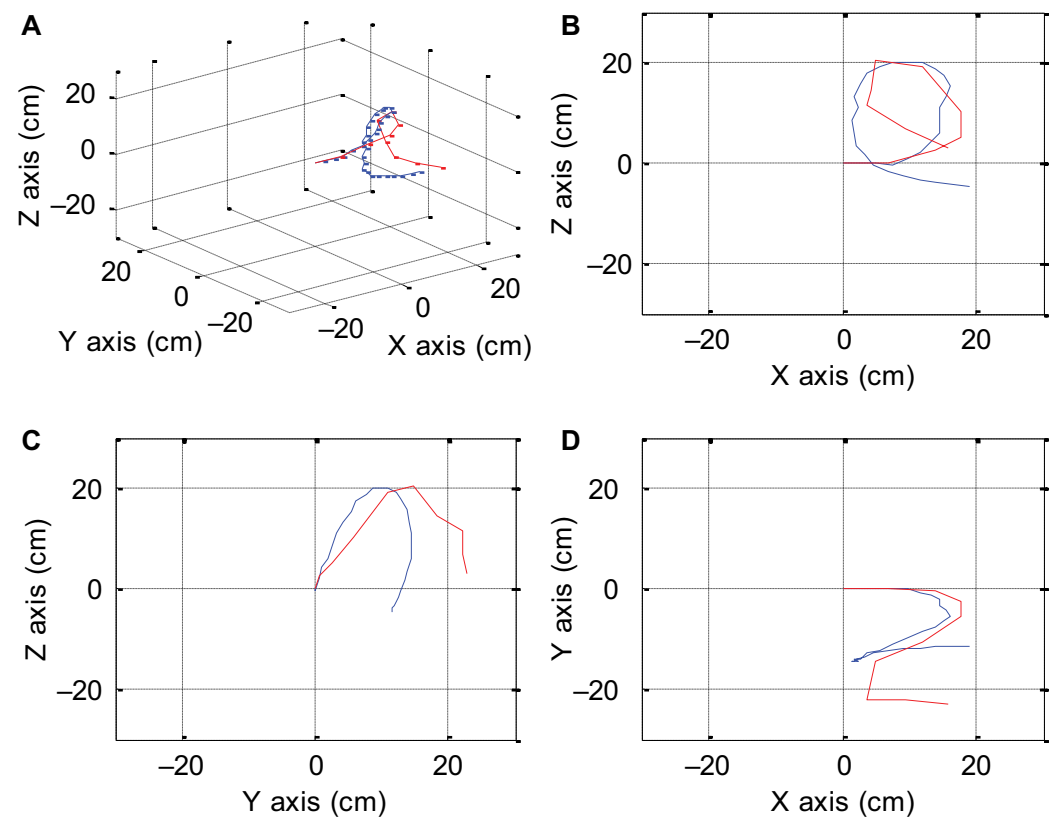

Figure 20 True alpha loop (red) versus generated alpha loop (blue) is shown. Isometric view (A), right-side view (B), left-side view (C), top view with right side as $\mathrm{X}$ axis (D).

\section{Reverse splenic flexure}

The reverse splenic flexure test was the only test with less desirable results. That withstanding, the predicted path still passes the visual test of comparison which is the most important factor. The experimental path is shown in Figure 25.

The model begins at the origin and travels in the positive $\mathrm{X}$ direction before traveling vertically in the $\mathrm{Z}$ direction. As it begins the ascent, the position in the $\mathrm{X}$ directions begins moving in the $-\mathrm{X}$ direction. Once the predicted path has reached the bottom of the flexure, it begins traveling laterally to the left in the $\mathrm{Y}$ direction and positively in both the $\mathrm{X}$ and $\mathrm{Z}$ directions. The predicted path shows a $17-\mathrm{cm}$ diameter tight loop with a difference in starting and finishing heights of $15 \mathrm{~cm}$. The true path is shown in Figure 26 .

In comparison, the true path shows a loop diameter of $20 \mathrm{~cm}$ with a slight spiral effect. The end of the initial loop is $8 \mathrm{~cm}$ left of the starting $\mathrm{Y}$ position, while in the predicted path, there was a $0 \mathrm{~cm}$ difference. The difference in starting 
to finishing height for the true path is shown to be $18 \mathrm{~cm}$ which is close that of the predicted path. Table 5 shows the angular deviation calculated for each corresponding vector for the reverse splenic flexure.

The overall average difference in vector angle was $79.27^{\circ}$. This is well above the allowable error in angular deviation. The highest difference was $81.80^{\circ}$, and the lowest was $77.24^{\circ}$. This represents a large variation between the expected and actual configurations. Vectors $3,6,7$, and 8 show a considerable difference that explains the differing shape to the end of the initial loop.

Table 2 The angles between vectors in degrees shown above

\begin{tabular}{llll}
\hline Vector & \multicolumn{3}{l}{ Alpha loop error } \\
\cline { 2 - 4 } & $\mathbf{I}$ & $\mathbf{2}$ & $\mathbf{3}$ \\
\hline 1 & 1.96 & 1.88 & 1.77 \\
2 & 17.87 & 23.05 & 25.64 \\
3 & 42.13 & 50.91 & 55.97 \\
4 & 7.82 & 8.78 & 19.85 \\
5 & 26.00 & 32.89 & 28.83 \\
6 & 22.62 & 24.21 & 21.08 \\
7 & 21.57 & 17.56 & 9.36 \\
8 & 54.44 & 49.36 & 37.77 \\
9 & 13.56 & 19.53 & 13.79 \\
10 & 16.14 & 10.99 & 16.55 \\
11 & 15.08 & 15.08 & 26.53 \\
Average & 21.7 & 23.11 & 23.4 \\
Total average & 22.7 & & \\
\hline
\end{tabular}

Notes: The average and total average for each trial are shown. The lowest average for the experiment is shown in yellow, the highest is in blue, and the overall average is in green.

\section{Discussion and conclusion}

After presenting all the data received from numerous experiments, the meaning of these results are discussed in this section starting with sensor calibration and initial prototyping and ending with the analysis of the loop formations.

\section{Sensor calibration}

The potentiometer has proven to be effective at measuring the angle of rotation. This can be seen from the calibration data in Figure 17. The potentiometer behaves linearly according to the equation $Y=-0.0239 X+5.4616$ from $20^{\circ}$ to $200^{\circ}$. The starting and final $\sim 20^{\circ}$ are nonlinear and thus are not used in the allowable angle of rotation. This means that the sensor can be used effectively to measure the angle from $-90^{\circ}$ to $+90^{\circ}$, covering $180^{\circ}$ of motion. From the initial two-dimensional (2D) test, it can be seen that the potentiometer is a viable option that can be used to measure the relative angle. It also verifies that the link system can be used to find the total position of the entire shaft as the generated image matched the real image almost perfectly. There is a maximum percent difference of $0.5 \%$ for the $2 \mathrm{D}$ test which is very low, showing that potentiometers can be very accurate.

\section{Initial prototype}

When the initial model was wired with sensors and integrated into the computer system, the generated model showed a very accurate fit to both original configurations. Even though the first test did not involve a sensor model enclosed inside of a
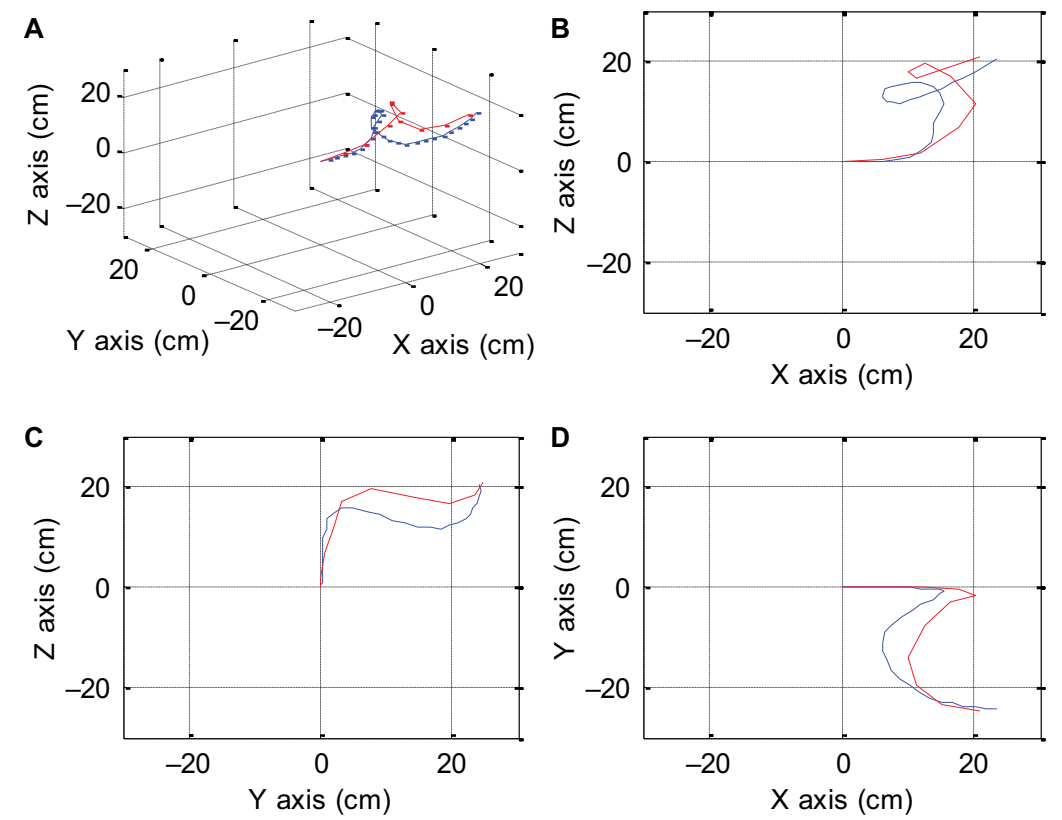

Figure 2 I Multiple views of the predicted sigmoid $\mathrm{N}$-spiral test are shown. Isometric view $(\mathbf{A})$, right-side view (B), left-side view (C), top view with right side as $\mathrm{X}$ axis (D). 

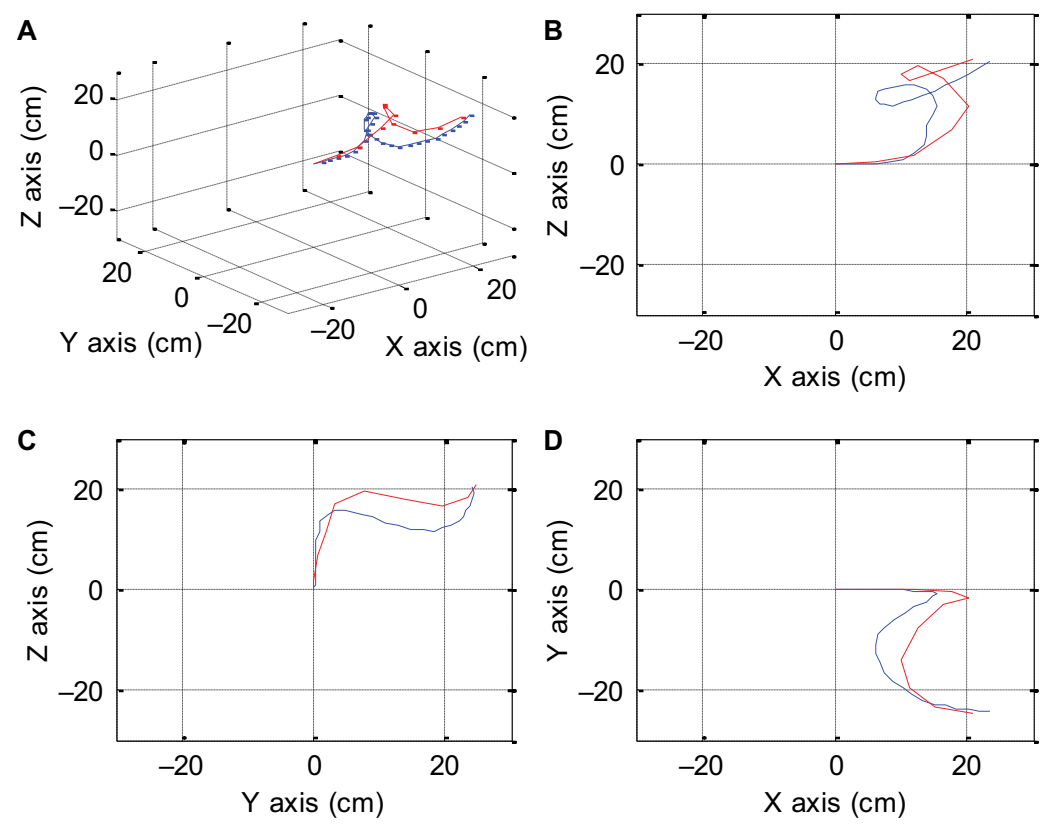

Figure 22 True N-loop (red) versus generated N-loop (blue) is shown. Isometric view (A), right-side view (B), left-side view (C), top view with right side as $\mathrm{X}$ axis (D).

Table 3 Vector angular deviation in degrees shown for each trial

\begin{tabular}{llll}
\hline Vector & \multicolumn{3}{l}{$\mathbf{N}$-spiral error } \\
\cline { 2 - 4 } & $\mathbf{I}$ & $\mathbf{2}$ & $\mathbf{3}$ \\
\hline 1 & 7.37 & 7.37 & 7.39 \\
2 & 13.04 & 12.94 & 10.83 \\
3 & 30.60 & 23.91 & 28.68 \\
4 & 24.89 & 17.18 & 22.53 \\
5 & 41.39 & 36.00 & 36.66 \\
6 & 33.39 & 35.96 & 32.25 \\
7 & 32.44 & 42.30 & 27.34 \\
8 & 26.06 & 27.16 & 27.07 \\
9 & 10.32 & 36.58 & 11.20 \\
10 & 19.04 & 27.21 & 11.54 \\
II & 23.50 & 26.12 & 16.24 \\
Average & 23.8 & 26.6 & 21.1 \\
Total average & 23.8 & & \\
\hline
\end{tabular}

Note: The total average is shown in green, whereas the lowest average is shown in yellow, and the highest average is shown in blue.

tube, the generated pictures still proved effective. The straight line image matched the setup perfectly. The second setup with the 3 -cm alpha loop matched very well. This shows that the prototype can accurately model the position of the shaft along a designated length. This in turn meant that a large model must be built to investigate the use of a colon replica and endoscope model. This model was developed and is the second prototype tested in this study.

\section{Looping analysis}

The looping analysis as previously stated shows great promise. Of all the loops, only one, the reverse splenic flexure, had difficulty showing the true configuration. Overall, it did show the presence of a splenic flexure loop but the accuracy of the loop size and location was less than optimal. This was believed to be because of a rotation of the sensor model inside of the endoscopic tube during the tight loop radius and abnormal loop shape.

The other three types of loop formations performed as predicted. Each test average showed $<25^{\circ}$ average deviation from the true path which was well below the maximum allowable error determined from Equation 18. This meant a very clear picture of the true loop that was being measured. All the loops passed the visual test indicating a loop had formed and that the scope needed to be worked with to relieve the loop formation. The first three tests clearly showed how the loop was formed and accurately measured its dimensions. The last test showed less favorable results but that indeed a loop was formed. The dimensions of the loop were close to the actual value but the location and direction of the endoscope tip were less accurate.

Overall, the most important factor concerning loop diagnosis is whether or not the loop is able to be identified as having been formed. The secondary factor is whether or not the loop can be identified by type. All the loops could be identified as having been formed, with the reverse splenic flexure having questionable results with loop type identification.

Some of the error that is associated with this method of error calculation comes from the measuring of the real configurations' $3 \mathrm{D}$ coordinates. The size of the colon tube (1.5 inches) and the size of the colonoscope tube ( 0.75 inches) contribute to the amount of error from the measurement of 
A

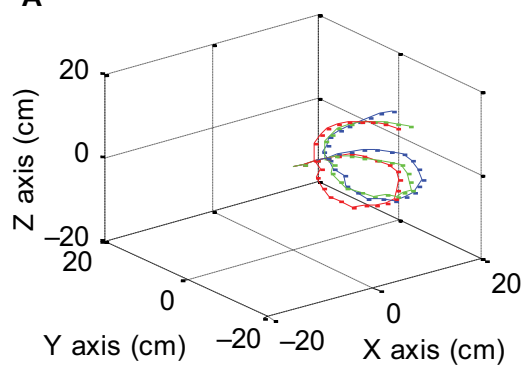

C

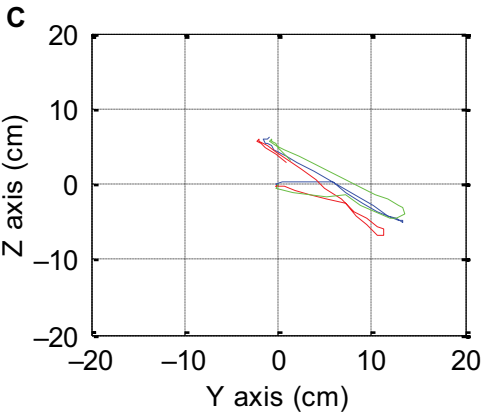

B

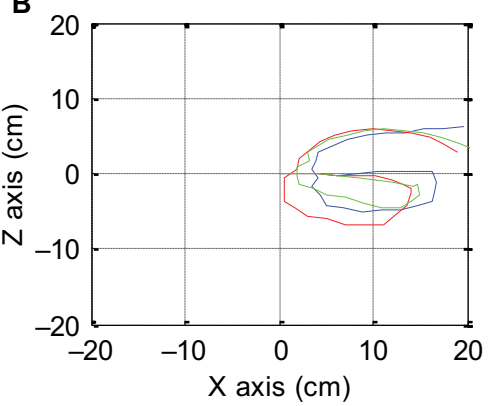

D

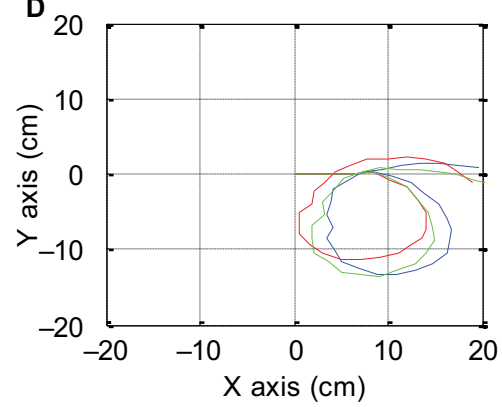

Figure 23 Multiple views of the predicted reverse gamma loop test are shown. Isometric view $(\mathbf{A})$, right-side view $(\mathbf{B})$, left-side view $(\mathbf{C})$, top view with right side as $\mathrm{X}$ axis (D).

A

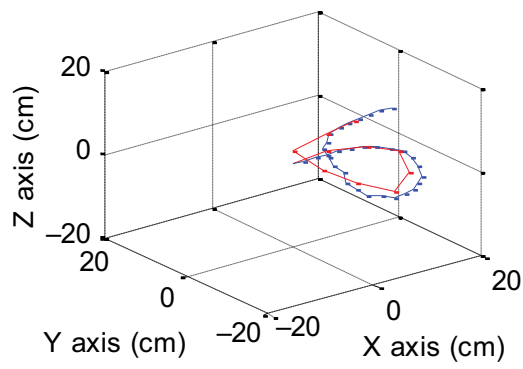

C

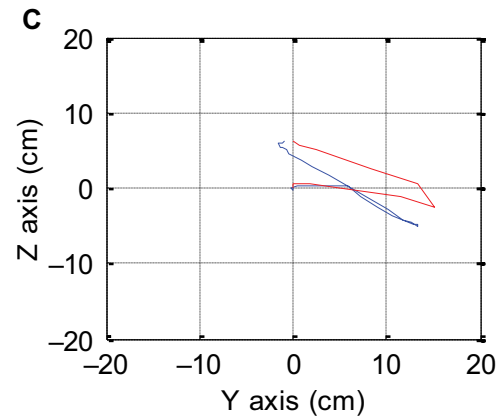

B

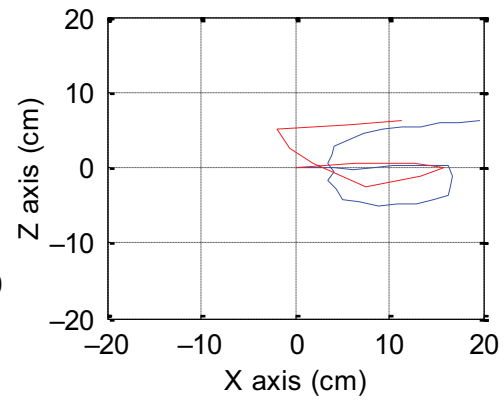

D

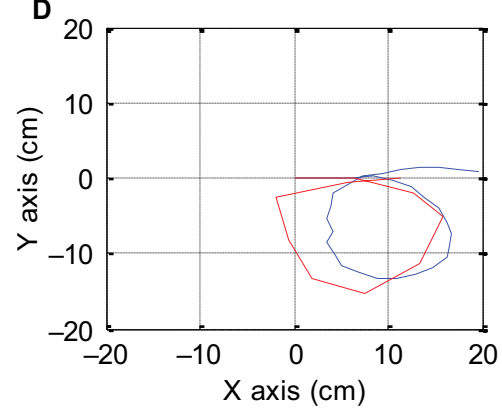

Figure 24 True reverse gamma loop (red) versus generated reverse gamma loop (blue) is shown. Isometric view (A), right-side view (B), left-side view (C), top view with right side as $\mathrm{X}$ axis (D).

the real coordinates. The path was chosen as the centerline of the colon model which could give a maximum deviation of 0.375 inches in a radial direction from the center of the colon tube. Error also occurs in the twisting of the sensor model inside of the colonoscope tube. The model was only fixed at the starting end of the loop. The end of the sensor model was free to rotate inside of the tube. This can cause additional error by shifting the reference planes for the $3 \mathrm{D}$ image. This is believed to have occurred in the reverse splenic flexure loop.

The comparisons between the true and the predicted path show that this method of loop formation detection can work in multiple different scenarios and proved its robustness. It showed that it can accurately detect many different types of loops of varying sizes and shapes. This adds to the benefit of having a loop detection device inside of the endoscope. 
The use of this model would have many benefits for the standard colonoscopy or endoscopic procedure. The causation of looping is a normal occurrence and can be very problematic. By using this device to accurately show the configuration of the endoscope during the procedure, the operator can more quickly, safely, and effectively navigate through the colon.

It has already been proven that if the colonoscopist knows the configuration of the endoscope shaft, operation times decrease, the number of incomplete operations decrease, and looping can more easily be handled. With the use of the

Table 4 Angular error values in degrees shown between vectors

\begin{tabular}{llll}
\hline Vector & \multicolumn{3}{l}{ Gamma loop error } \\
\cline { 2 - 4 } & $\mathbf{1 . 0 0}$ & $\mathbf{2 . 0 0}$ & $\mathbf{3 . 0 0}$ \\
\hline 1 & 7.39 & 7.41 & 7.40 \\
2 & 7.75 & 6.81 & 10.24 \\
3 & 10.05 & 25.13 & 19.23 \\
4 & 36.08 & 37.70 & 19.62 \\
5 & 7.29 & 31.64 & 31.44 \\
6 & 30.49 & 16.06 & 15.47 \\
7 & 23.77 & 46.75 & 37.38 \\
8 & 14.36 & 37.59 & 32.71 \\
9 & 22.42 & 11.27 & 7.00 \\
10 & 1.59 & 20.93 & 21.70 \\
$I I$ & 6.40 & 44.85 & 32.09 \\
Average & 15.2 & 26.0 & 21.3 \\
Total average & 20.8 & & \\
\hline
\end{tabular}

Notes: The total average error is shown in green. The lowest average error for a single trial is in yellow, whereas the highest is in blue. potentiometer system, the configuration can be accurately defined during procedures. This will give the doctor the ability to counteract looping and lead to a more efficient and safer procedure.

\section{Hardware and software development}

The model needs to be smaller in order to fit inside of the colonoscope. There do exist some small potentiometers that are meant for only a short number of turns to the axle. This could be a potentially useful sensor upgrade as the size of the sensor is dramatically smaller. That being said, it is also not as reliable as a medical device. With future technology improving, potentiometers are growing smaller but are just as reliable. Future smaller sensors could be used in place of the ones that are currently in place for this model. The link design must also be redesigned to accommodate the smaller array of sensors as well. This could be problematic as the smaller the links are, the more likely they are to fail under similar loads.

The operating program for the 3D vector image generation is currently MATLAB. The voltage values are taken from the Signal Express program and exported to an Excel file where it is then referenced from the MATLAB program. Future work could involve simultaneous uploading straight from the Signal Express program directly into the MATLAB file. Another option would be to choose a different program to generate the 3D images and videos. This could be done using the LabVIEW program.
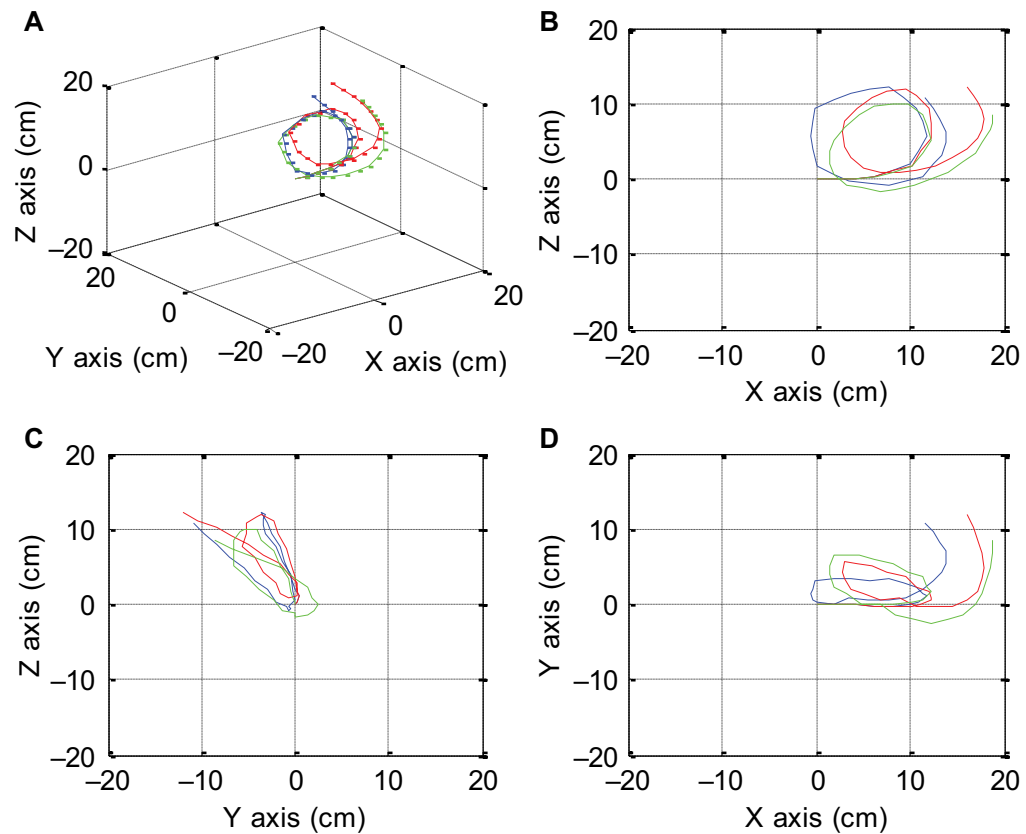

Figure 25 Multiple views of the predicted reverse splenic flexure test are shown. Isometric view (A), right-side view (B), left-side view (C), top view with right side as $X$ axis (D). 

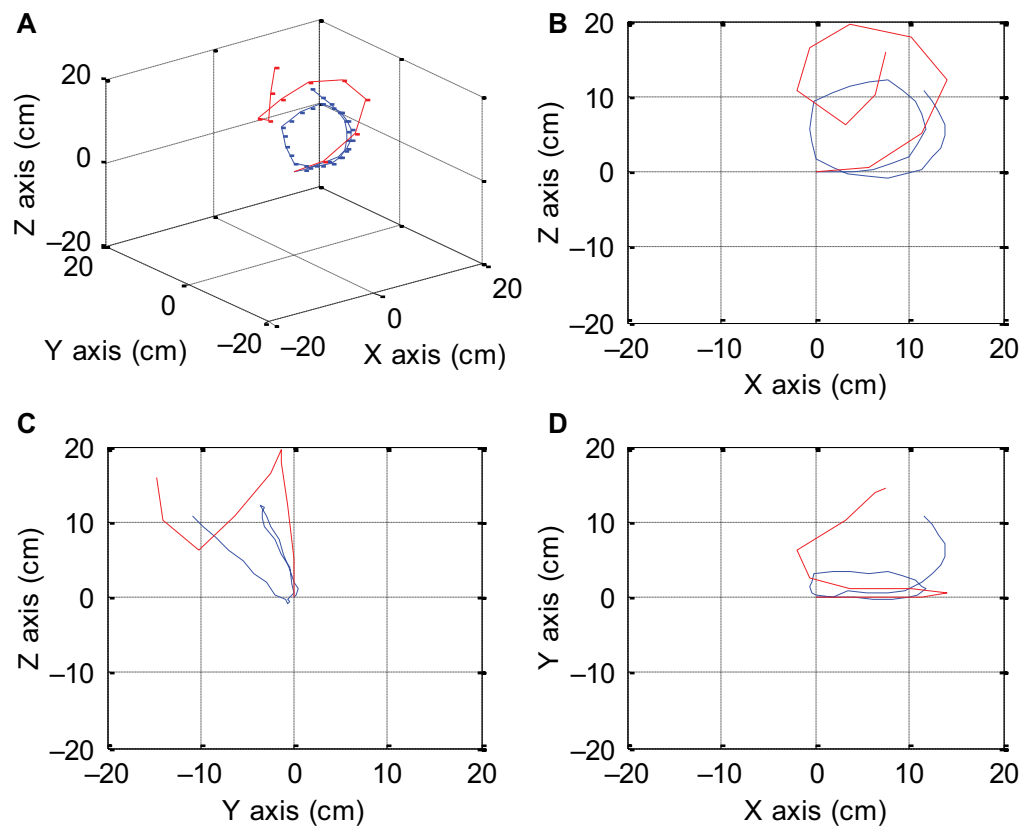

Figure 26 True reverse splenic flexure (red) versus generated reverse splenic flexure (blue) is shown. Isometric view (A), right-side view (B), left-side view (C), top view with right side as $X$ axis (D).

Table 5 Angular deviation in degrees between vectors shown with the total average in green

\begin{tabular}{llll}
\hline Vector & \multicolumn{3}{l}{ Reverse splenic flexure error } \\
\cline { 2 - 4 } & $\mathbf{1 . 0 0}$ & $\mathbf{2 . 0 0}$ & $\mathbf{3 . 0 0}$ \\
\hline 1 & 4.33 & 4.30 & 4.12 \\
2 & 43.16 & 38.92 & 39.41 \\
3 & 113.20 & 103.76 & 117.37 \\
4 & 83.08 & 100.32 & 92.88 \\
5 & 34.19 & 54.95 & 51.52 \\
6 & 119.95 & 123.70 & 117.71 \\
7 & 119.27 & 132.63 & 144.57 \\
8 & 98.98 & 101.10 & 113.61 \\
9 & 38.96 & 44.68 & 28.99 \\
10 & 83.01 & 85.64 & 65.51 \\
11 & 111.54 & 109.76 & 90.76 \\
Average & 77.24 & 81.80 & 78.77 \\
Total average & 79.27 & & \\
\hline Note: The higest & &
\end{tabular}

Note: The highest singular trial average is in blue and the lowest is in yellow.

One aspect that is not discussed in this research is the orientation or pose of the model along the length of the colonoscope shaft. This was not a major concern because the most important point regarding this work was the overall shape of the colonoscope. If the shape was known, the orientation did not matter. In the future, knowing the orientation of the colonoscope shaft could provide additional benefit by giving the amount of twisting along the shaft length. This could be useful if paired with loop detection and prevention discussed below.
The next step involves detecting the loops before they form and warning the doctor. Once a loop has formed to $50 \%$ or has formed a half circle, the doctor would be given a warning message saying that a loop was forming. It would also inform the operator of certain maneuvers that could be done to the scope depending on the type of loop formation. ${ }^{22}$ If the orientation was added to this, the amount and direction of the rotation could help identify the loops as they formed. Loop detection could be carried out by testing a number of additional loops to form a database of loop configurations. Then, as the model is pushed into the loop, it would analyze the current configuration compared with the different known loops to tell the operator exactly what type of loop had formed. Along with this would be a series of twists and corrections that need to be made to the scope to counteract and prevent the loop from forming further. ${ }^{23}$

\section{Disclosure}

The authors report no conflicts of interest in this work.

\section{References}

1. Korman LY, Overholt BF, Box T, Winker CK. Perforation during colonoscopy in endoscopic ambulatory surgical centers. Gastrointest Endosc. 2003;58(4):554-557.

2. Shah SG, Saunders BP, Brooker JC, Williams CB. Magnetic imaging of colonoscopy: an audit of looping, accuracy and ancillary maneuvers. Gastrointest Endosc. 2000;52(1):1-8.

3. Helmut $\mathrm{M}$, editor. Inserting the endoscope and advancing it in the colon. In: Atlas of Colonoscopy: Techniques, Diagnosis, Interventional Procedures. New York: Thieme Stuttgart; 2004:28. 
4. Helmut M, editor. Polyps and polyposis syndromes. In: Atlas of Colonoscopy: Techniques, Diagnosis, Interventional Procedures. New York: Thieme Stuttgart; 2004:73-75.

5. Helmut M, editor. General information regarding examination. In: Atlas of Colonoscopy: Techniques, Diagnosis, Interventional Procedures. New York: Thieme Stuttgart; 2004:5.

6. Kamal A, Van Dam J. Reduced loop formation using a computerassisted colonoscope (the NeoGuide system) compared with standard colonoscopy. Gastrointest Endosc. 2006;63(5):AB228.

7. Eickhoff A, Van Dam J, Jakobs R, et al. Computer-assisted colonoscopy (the NeoGuide endoscopy system): results of the first human clinical trial ("PACE study"). Am J Gastroenterol. 2007;102(2):261-266.

8. Van Dam J, Eickhoff A, Jakobs R, Kudis V, Hartmann D, Riemann JF. Computer-assisted colonoscopy (the NeoGuide system): results of the first human clinical trial. Gastrointest Endosc. 2006;63(5):AB100.

9. Raju GS, Rex DK, Kozarek RA, Ahmed I, Brining D, Pasricha PJ. A novel shape-locking guide for prevention of sigmoid looping during colonoscopy. Gastrointest Endosc. 2004;59(3):416-419.

10. Friedland S, Soetikno RM. Small caliber overtube-assisted colonoscopy. World J Gastroenterol. 2007;13(44):5933-5937.

11. Friedland S, Soetikno RM. Small caliber overtube-assisted colonoscopy in patients with incomplete colonoscopy using standard endoscopes. Gastrointest Endosc. 2007;65(5):AB113.

12. Striegel J, Jakobs R, Van Dam J, Weickert U, Riemann JF, Eickhoff A. Determining scope position during colonoscopy without use of ionizing radiation or magnetic imaging: the enhanced mapping ability of the NeoGuide endoscopy system. Surg Endosc. 2011;25(2):636-640.

13. Coderre S, Anderson J, Rikers R, Dunckley P, Holbrook K, McLaughlin $\mathrm{K}$. Early use of magnetic endoscopic imaging by novice colonoscopists: improved performance without increase in workload. Can J Gastroenterol. 2010;24(12):727.
14. Kobayashi T, Leung AK, Hutchins SW. Design of a manual device to measure ankle joint stiffness and range of motion. Prosthet Orthot Int. 2011;35(4):478-481.

15. Chen Q, Lazennec JY, Guyen O, Kinbrum A, Berry DJ, An KN. Technical note: validation of a motion analysis system for measuring the relative motion of the intermediate component of a tripolar total hip arthroplasty prosthesis. Med Eng Phys. 2005;27(6):505-512.

16. O’Donovan KJ, Kamnik R, O’Keeffe DT, Lyons GM. An inertial and magnetic sensor based technique for joint angle measurement. J Biomech. 2007;40(12):2604-2611.

17. Perry JC, Rosen J. Design of a 7 degree-of-freedom upper-limb powered exoskeleton. In: The First IEEE/RAS-EMBS International Conference on Biomedical Robotics and Biomechatronics, 2006. BioRob 2006; February 20-22, 2006:805-810; Pisa, Italy.

18. Mao Y, Agrawal SK. Design of a cable-driven arm exoskeleton (CAREX) for neural rehabilitation. IEEE Trans Robot. 2012;28(4):922-931.

19. Yu Y, Narita Y, Harada Y, Nakao T. Research of 3-DOF active rotational ball joint. In: IEEE/RSJ International Conference on Intelligent Robots and Systems, 2009. IROS 2009; October 10-15, 2009:5153-5158; St Louis, MO, USA.

20. Cheng WB, Moser MAJ, Kanagaratnam S, Zhang WJ. Analysis of and mathematical model insight into loop formation in colonoscopy. Proc Inst Mech Eng H. 2012;226(11):858-867.

21. Sato K, Fujinuma S, Sakai Y. Evaluation of the looping formation and pain during insertion into the cecum in colonoscopy. Digest Endosc. 2006;18(3):181-187.

22. JungHun Choi and David Drozek. Detection of the looping in colonoscopy using bending sensors. Open Med Devices J. 2012;3:1-7.

23. Church JM. Ancillary colonoscope insertion techniques. Surg Endosc. 1993;7(3):191-193.
Medical Devices: Evidence and Research

\section{Publish your work in this journal}

Medical Devices: Evidence and Research is an international, peerreviewed, open access journal that focuses on the evidence, technology, research, and expert opinion supporting the use and application of medical devices in the diagnosis, monitoring, treatment and management of clinical conditions and physiological processes. The identification of novel

\section{Dovepress}

devices and optimal use of existing devices which will lead to improved clinical outcomes and more effective patient management and safety is a key feature. The manuscript management system is completely online and includes a quick and fair peer-review system. Visit http://www. dovepress.com/testimonials.php to read real quotes from authors. 